

\title{
L'économie circulaire : cercle vertueux ou cercle vicieux ?Le cas de l'utilisation de terres maraîchères pour aménager des espaces verts urbains
}

Clément Gitton, Yoann Verger, Florence Brondeau, Ronald Charvet, François Nold, Philippe Branchu, Francis Douay, Isabelle Lamy, Christian Mougin, Caroline Petit, et al.

\section{To cite this version:}

Clément Gitton, Yoann Verger, Florence Brondeau, Ronald Charvet, François Nold, et al.. L'économie circulaire : cercle vertueux ou cercle vicieux ?Le cas de l'utilisation de terres maraîchères pour aménager des espaces verts urbains. Géocarrefour - Revue de géographie de Lyon, 2018, 92 (92), 10.4000/geocarrefour.11950 . hal-01972726

\section{HAL Id: hal-01972726 \\ https://hal.science/hal-01972726}

Submitted on 26 May 2020

HAL is a multi-disciplinary open access archive for the deposit and dissemination of scientific research documents, whether they are published or not. The documents may come from teaching and research institutions in France or abroad, or from public or private research centers.
L'archive ouverte pluridisciplinaire HAL, est destinée au dépôt et à la diffusion de documents scientifiques de niveau recherche, publiés ou non, émanant des établissements d'enseignement et de recherche français ou étrangers, des laboratoires publics ou privés. 


\section{Géocarrefour}

$92 / 2 \mid 2018$

Visibilité et invisibilité de la pollution des sols dans les territoires (post)industriels : de nouvelles perspectives sur la résilience et la justice environnementale?

\section{L'économie circulaire : cercle vertueux ou cercle vicieux ?Le cas de l'utilisation de terres maraîchères pour aménager des espaces verts urbains}

The circular economy: vicious or virtuous circle? The case of vegetable gardens used to develop green spaces

Clément Gitton, Yoann Verger, Florence Brondeau, Ronald Charvet, François Nold, Philippe Branchu, Francis Douay, Isabelle Lamy, Christian Mougin, Caroline Petit et Elisabeth Rémy

\section{OpenEdition}

\section{Journals}

Édition électronique

URL : http://journals.openedition.org/geocarrefour/11950

DOI : $10.4000 /$ geocarrefour. 11950

ISSN : 1960-601X

Éditeur

Association des amis de la Revue de géographie de Lyon

Ce document vous est offert par INRA Institut National de la Recherche Agronomique

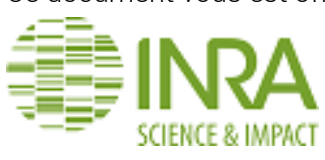

Référence électronique

Clément Gitton, Yoann Verger, Florence Brondeau, Ronald Charvet, François Nold, Philippe Branchu, Francis Douay, Isabelle Lamy, Christian Mougin, Caroline Petit et Elisabeth Rémy, «L'économie circulaire : cercle vertueux ou cercle vicieux ? Le cas de l'utilisation de terres maraîchères pour aménager des espaces verts urbains », Géocarrefour [En ligne], 92/2 | 2018, mis en ligne le 28 décembre 2018, consulté le 12 janvier 2019. URL : http://journals.openedition.org/geocarrefour/11950 ; DOI : 10.4000/geocarrefour. 11950

Ce document a été généré automatiquement le 12 janvier 2019.

(C) Géocarrefour 


\section{L'économie circulaire : cercle vertueux ou cercle vicieux ?Le cas de l'utilisation de terres maraîchères pour aménager des espaces verts urbains}

The circular economy: vicious or virtuous circle?The case of vegetable gardens used to develop green spaces

Clément Gitton, Yoann Verger, Florence Brondeau, Ronald Charvet, François Nold, Philippe Branchu, Francis Douay, Isabelle Lamy, Christian Mougin, Caroline Petit et Elisabeth Rémy

\section{Introduction}

1 Depuis plusieurs années, les collectivités travaillent à satisfaire les nouveaux besoins et usages qui se créent dans les espaces verts urbains. Auparavant les pelouses restaient inaccessibles aux publics et les usages se bornaient à un agrément visuel et de promenade. Aujourd'hui, ces espaces sont ouverts et sont devenus des espaces récréatifs où peuvent se côtoyer des jeux d'enfants, des espaces de biodiversité, des espaces de détente, d'agriculture ou de jardinage collectif. C'est dans ce contexte que la question de la qualité des sols est devenue centrale par rapport à ces nouveaux usages.

2 La plupart des collectivités ont donc travaillé sur la qualité des sols pour statuer sur leur compatibilité avec la culture de végétaux consommables. Puis, c'est dans le cadre de la prévention du saturnisme infantile que le Haut Conseil de la Santé Publique (HCSP) a fixé en 2014 des seuils de gestion liés aux concentrations en plomb dans les sols, ce qui a initié une nouvelle mise en visibilité des risques associés à la pollution des sols urbains. En effet, au regard des risques liés à l'ingestion de particules de terre liée au portée main-bouche 
pour les enfants de moins de 6 ans, un seuil de vigilance de $100 \mathrm{mg}$ de plomb par $\mathrm{kg}$ de terre a été proposé pour les sols des espaces verts (EV). Suite à la parution de ces nouveaux objectifs de gestion, de nombreuses collectivités ont amorcé un travail de caractérisation de ces sols.

C'est le cas pour la Ville de Paris qui possède environ 500 hectares d'espaces verts (hors bois de Vincennes et de Boulogne) aménagés depuis plus de 200 ans. Tous ces EV ont reçu lors de leur création un apport de terre dite "végétale " ${ }^{1}$ pour favoriser un développement horticole. Bien que la terre végétale d'apport fasse l'objet de contrôle de qualité depuis 1982, il est apparu que la Ville de Paris (comme la plupart des collectivités en France) n'avait pas encore une parfaite connaissance de sa qualité et de sa traçabilité.

4 S'appuyant sur des travaux conjoints entre le laboratoire d'agronomie de la Ville de Paris ainsi que l'Agence d'Écologie Urbaine, les Universités (Paris IV, Paris VII, Paris-Saclay) et les laboratoires associés (UMR 8185 ENeC, UMR 1048 INRA-Sad-Apt), deux stages de Master ont été menés en parallèle. Le premier s'est intéressé à l'évolution des mesures de caractérisation des sols et de leur qualité (Canolle, 2016), et le second (Gitton, 2016) a été davantage orienté vers l'histoire des espaces verts et de l'environnement urbain parisien. Pour répondre à la question complexe que pose notre sujet, il a été décidé d'ouvrir l'encadrement de ces deux étudiants à divers organismes de recherches (Cerema, INRAVersailles, ISA Lille), offrant un travail interdisciplinaire à l'interface entre l'agronomie, l'écotoxicologie, la géochimie, la géographie et la sociologie.

5 Le présent texte, destiné à mettre en évidence une partie des résultats obtenus, a pour ambition de poser un certain nombre de questions quant à la qualité des sols urbains et à leur capacité à être un support d'activités récréatives et/ou de production alimentaire à l'heure où les efforts pour passer à une économie circulaire aboutissent à privilégier les circuits courts et l'approvisionnement alimentaire local (Bonnefoy et Brand, 2014). En effet, de nombreuses villes s'engagent pour mettre en place des formes de gouvernance des systèmes alimentaires ${ }^{2}$. En France, la loi d'avenir pour l'agriculture, l'alimentation et la forêt de 2014 prévoit l'élaboration de plans alimentaires territoriaux qui doivent permettre de rapprocher production et consommation locales. Si l'accent est principalement mis sur le développement des filières courtes, certains acteurs envisagent aussi la remise en culture de terrains en ville (friches, trames vertes, espaces verts, etc.). Des spécialistes de l'agriculture urbaine (Duchemin, 2013) comme certaines collectivités locales ${ }^{3}$ soutiennent l'idée «de nourrir la ville par la ville $»^{4}$; d'autres développent le concept de «villes vivrières" (Blanc, Dubost, Lizet, 2014), via la relocalisation des cultures alimentaires en milieu intra-urbain. Dès lors, il s'agit de s'interroger sur les caractéristiques et la qualité des terres apportées qui ont transité et qui transitent encore de la proche banlieue vers les centres urbains pour l'aménagement des espaces verts et des nombreux potagers urbains qu'ils accueillent. Dans ce contexte, il n'est pas inutile de réinterroger les principes et les rouages complexes de l'économie circulaire au regard des flux de terres végétales (qui constituent le support de ces espaces verts, dont les jardins) dans une démarche à la fois historique et spatiale.

6 Le concept d'économie circulaire, envisagé dès le milieu des années 1960 (Boulding, 1966), puis formulé par deux économistes de l'environnement (Pearce et Turner, 1989), avant d'être repris par les promoteurs du courant de l'écologie industrielle (Frosch et Gallopoulos, 1989; Erkman, 1997) a été popularisé en France (Lévy, 2009) lors des discussions liées au Grenelle 2 de l'environnement (Gueymard et al., 2015). L'ADEME le définit comme "un système économique d'échange et de production qui, à tous les stades du 
cycle de vie des produits (biens et services), vise à augmenter l'efficacité de l'utilisation des ressources et à diminuer l'impact sur l'environnement tout en développant le bien être des individus» (ADEME, 2018, p. 2). L'économie circulaire recouvre une diversité de thèmes ayant en commun la sobriété des ressources et l'établissement de boucles de recyclage ${ }^{5}$. Les travaux scientifiques qui envisagent la relation entre la nécessaire mise à l'écart des flux et des stocks de polluants et l'économie circulaire en milieu urbain ne sont pas légion (Newell, Cousins, 2014). Dans le champ de l'écologie industrielle, seules quelques rares voix se font entendre sur le problème de la contamination des flux (Baxter, Aurisicchio, Childs, 2017 ; Zhu et al., 2017) et de la nécessaire extraction des déchets dangereux de la boucle. Augustsson (2017) montre notamment que l'arsenic utilisé avant 2003 pour protéger les bois de construction en Suède pollue toujours les flux de recyclage du bois.

Cet article se veut une modeste contribution à cette mise en débat visant à s'interroger sur certaines limites de l'économie circulaire en mettant en évidence les risques de transfert de polluants via les flux de terres végétales d'apports ayant servi à constituer les espaces verts. L'hypothèse est la suivante: les terres maraîchères polluées aux périphéries de la ville par les activités humaines dès le XIX ${ }^{\mathrm{e}}$ siècle ont-elles contribué à la contamination des sols des EV en éléments traces métalliques (dont le plomb) lors de leur import au cœur de Paris pour réaliser les parcs et jardins?

Ces pistes de recherche seront appuyées par des mesures sur le terrain, élaborées dans le cadre d'un protocole précis (Canolle, op. cit.; Gitton, op. cit.). La démonstration s'attachera d'abord à reconstituer l'histoire chronologique et spatiale de l'aménagement des espaces verts parisiens intra-muros.

\section{La contamination des sols des EV en milieu urbain : hypothèses et choix des sites}

\section{Les espaces verts parisiens : définition et repères historiques}

Clarifier brièvement la définition du terme " espaces verts » apparaît comme un préalable indispensable. D'après le Centre d'études sur les réseaux, les transports, l'urbanisme et les constructions publiques (CERTU, 2001, p. 73), il s'agirait de " tous les espaces végétalisés, arborés ou non, qui prennent place dans le tissu urbain. Qu'ils soient publics ou privés, ouverts ou fermés aux habitants, les espaces verts ménagent une discontinuité végétale entre les zones bâties et les annexes minéralisées. Les EV composent un maillage interstitiel de verdure (espace libre) et ils se définissent par opposition aux espaces construits (espace plein) ». Deux autres paramètres sont parfois introduits, à savoir la « superficie » et la « fonction » (Corvol, 1994).

On peut donc considérer que les espaces verts comprennent des jardins, des parcs, des squares, des établissements sportifs, des promenades, des places, des jardins sur dalle, des jardins d'immeubles ainsi que des pelouses ouvertes (des esplanades comme le Champ de Mars) ou des pelouses «fermées » (prairies) (Gitton, op. cit.). C'est au XIX siècle, et plus précisément dans sa seconde moitié, que Paris voit éclore une multitude de parcs, jardins et squares sous l'influence de Louis Napoléon Bonaparte.

11 Afin de rendre compte de l'évolution d'ouverture d'espaces verts durant le XIXe siècle et jusqu'à aujourd'hui, une base de données du Service d'Exploitation des Jardins au sein de la Direction des Espaces Verts et de l'Environnement (DEVE), recensant tous les EV parisiens, a été mobilisée corrélativement à la typologie historique élaborée par Jean 
Christophe Lucas, conférencier à la Ville de Paris (2015). Celle-ci reprend en partie les « grandes périodes historiques » de l'histoire française contemporaine comme le «Second Empire " mais également des périodes plus ou moins longues correspondant à des conceptions urbanistiques et paysagères bien particulières. Ainsi, la figure $1^{6}$ met en évidence les différentes phases chronologiques de la mise en place des EV publics parisiens et leurs évolutions. Le véritable point de départ s'effectue sous le Second Empire avec la création de $25 \mathrm{EV}$ contre seulement 4 pour la période précédente. Cela s'explique par l'absorption en 1860 de communes suburbaines comme Charonne, Belleville, Vaugirard, Passy, etc., augmentant alors le nombre d'arrondissements de 12 à 20 . Pour les deux périodes qui suivent, on constate une accélération dans le rythme de réalisation d'EV et une véritable « explosion » des ouvertures après la Seconde Guerre mondiale (241) qui semble se poursuivre dans la période récente : 68 pour la dernière période de 2000 à 2016. Toutefois, à travers la surface totale d'EV créée par période, on observe une diminution de la superficie moyenne attribuée aux nouveaux EV, à l'exception du «renouveau des années $1930 »^{7}$.

Figure 1 : Évolution du nombre de création d'espaces verts et de la surface totale (ha) en fonction de la typologie historique de Jean Christophe Lucas (sans les bois de Vincennes et de Boulogne)

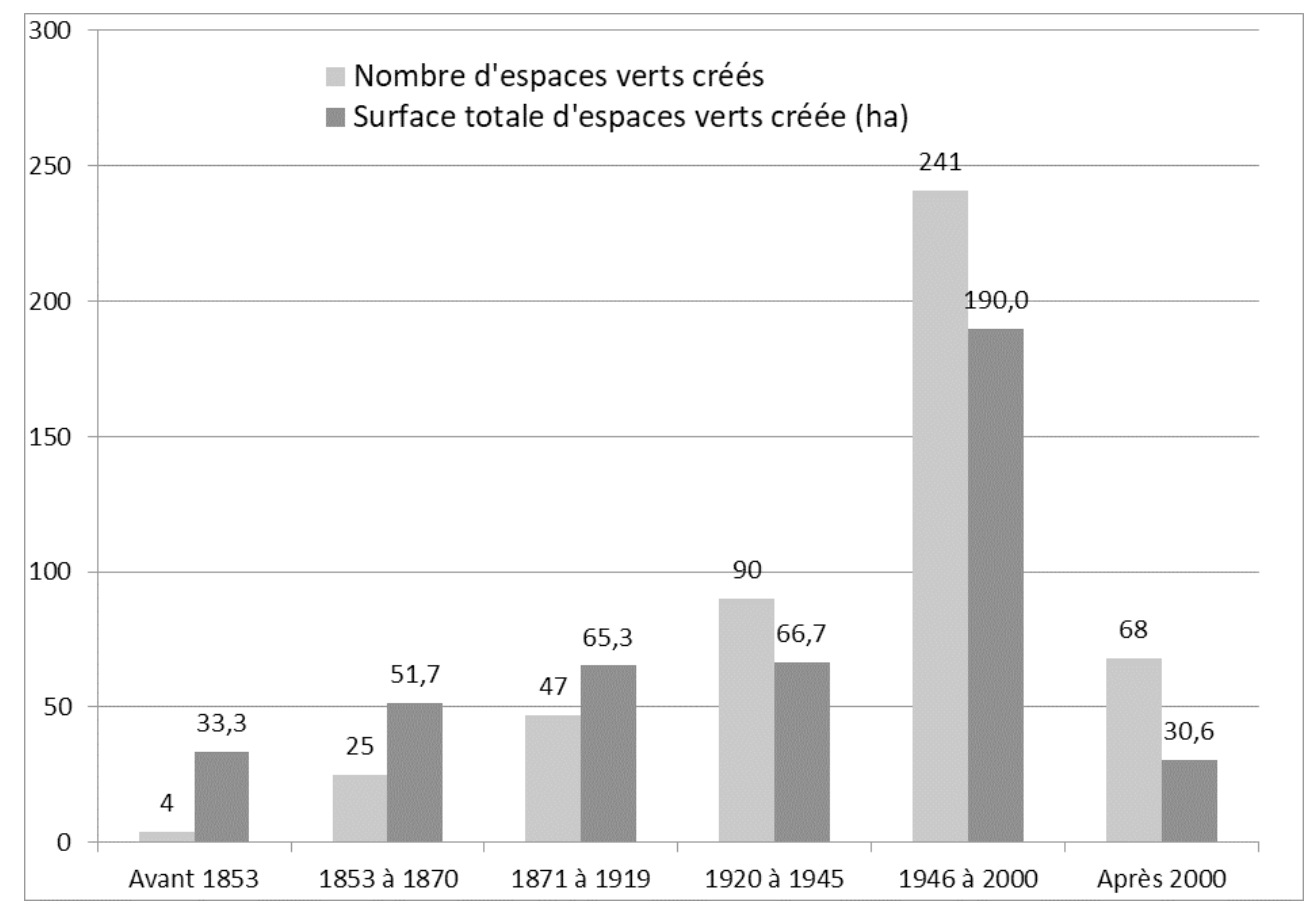

Source : Gitton, op. cit.

12 Les créations d'EV posent question en termes de qualité des sols. En effet, depuis la fin de la Seconde Guerre mondiale et jusqu'à nos jours, ils sont réalisés sur des espaces vacants, des délaissés urbains et/ou des friches comme les anciens terrains industriels (par exemple, le parc André Citroën) ou encore sur des zones ferroviaires non utilisées ou exploitées par le Réseau Ferré de France (parc Clichy-Batignolles, parc de Bercy, Jardin d'Eole, aménagement sur certaines zones de la petite ceinture non exploitée pour le transport des marchandises depuis 1993 et des voyageurs depuis 1934). Ces dernières années, la grande majorité des EV sont créés dans le cadre d'un processus de végétalisation de la voie publique et de verdissement de la ville (jardinets, talus, platesbandes, etc.). Un certain engouement pour les jardins partagés depuis le début des années 
2000 (plus d'une centaine créée) ${ }^{8}$ y contribue également. Un responsable du laboratoire d'agronomie de la ville de Paris précise ${ }^{9}$ qu'un apport en «terre végétale » est effectué systématiquement in situ lors de la création d'un nouvel espace vert.

\section{Hypothèses quant aux sources de contamination des sols des EV : des pistes de réflexion issues de recherches historiques}

13 La provenance de ces terres végétales d'apport est primordiale pour comprendre le degré de contamination potentiel des terres. Vraisemblablement, selon l'enquête réalisée au laboratoire d'agronomie de la Ville de Paris en 2016, la « terre végétale » des EV parisiens est partiellement issue des terrains maraîchers d'île-de-France. Selon les périodes, elle proviendrait d'abord de Paris, puis de la petite couronne (Gaulin, 1987) et, à l'heure actuelle, des terrains agricoles de la grande couronne. De ce fait, au regard des techniques de fertilisation utilisées par les maraîchers au XIXe siècle et jusque dans l'Entre-DeuxGuerres (Barles, 1999, 2002), il est tout à fait envisageable que les terres végétales aient été contaminées avant même d'arriver sur les sites des EV. En effet, «au début du XX siècle, on souligne encore le rôle capital qu'ont joué les boues de Paris pour la fertilisation du département de la Seine aux terres naturellement peu productives [...]» (Barles, 2002, p. 146). Dès le XIX ${ }^{e}$ siècle des pratiques culturales sont aussi sujettes à débat: "Les jardiniersmaraîchers [...] ne connaissaient pour engrais et stimulant que le fumier, l'eau et la chaleur ; rien de plus. Plusieurs d'entre nous se rappellent, avoir vu employer les balayures des rues de Paris ${ }^{10}$ pour améliorer la terre de certains marais; mais cet usage est abandonné depuis longtemps par les maraichers dans l'enceinte de la capitale, quoiqu'il soit toujours suivi, et avec raison, dans la petite culture des environs » (Moreau, Daverne, 1845, p. 26) ${ }^{11}$.

14 Les boues de Paris étaient constituées de résidus urbains très divers, aussi bien de résidus végétaux, d'ordures ménagères, de boues de terre, que les ramassés des rues, les matières fécales tirées des fosses d'aisances - voirie de Montfaucon pour Paris au début du XIX ${ }^{\mathrm{e}}$ siècle, puis de Bondy en Seine Saint-Denis une fois le canal de l'Ourcq percé (Barles, 1999). A la fin du XIX siècle, les eaux usées de la capitale commencent à être épandues dans les champs de communes proches comme à Gennevilliers ou Achères, où les sols sableux épurent efficacement tout en générant des rendements maraîchers élevés. L'accumulation des éléments traces métalliques (ETM) dans les sols sera mise en évidence (notamment en plaine d'Achères et de Pierrelaye) et la pratique des champs d'épandage interdite (Mandinaud, 2005). Depuis les années 1970, avec la croissance démographique et l'augmentation du nombre de stations d'épuration, la production des boues de station augmente de façon continue. Celles-ci sont principalement épandues en agriculture ${ }^{12}$ (2,5\% de la surface agricole nationale est concernée par ces épandages $)^{13}$. L'épandage agricole est considéré comme la solution la plus économique, car les coûts sont plus faibles que l'incinération ou la mise en décharge, et la plus pertinente sur le plan agronomique, car considéré comme un fertilisant organique substituable aux engrais chimiques de synthèse. Demeurent toutefois des interrogations sur le plan environnemental (Lupton, 2011), que nous développerons plus loin.

On sait que les apports de plomb anthropique ont été considérables en France depuis le $\mathrm{XIX}^{\mathrm{e}}$ siècle : " Un tiers de ces apports (13 millions de tonnes d'après Lestel, 2002) a eu lieu avant 1914, un petit quart pendant l'Entre-deux guerres [...]. Ce stock très urbain, est actuellement localisable dans différents compartiments de l'anthroposphère: dans des produits finis en cours d'utilisation (canalisations, peintures...), dans les objets mis au rebus, dans l'air, l'eau et le sol, si 
bien que la situation actuelle reflète moins la production présente de ce métal que les usages passés . [...] De même une récente étude portant sur le métabolisme du mercure à Stockholm montre-t-elle que les apports, après avoir cru jusque dans les années 1970, n'ont cessé de diminuer depuis [...] Mais les stocks, eux, sont bien là, en particulier dans les sédiments (Barles, 2002, p. 146).

On peut donc raisonnablement envisager qu'au XIX ${ }^{e}$ siècle, les boues de Paris épandues dans les zones maraîchères qui servent d'approvisionnement aux circuits de terres végétales contenaient également une part significative d'éléments traces métalliques (ETM) : " Dès le XIXe siècle, on oublie ces interfaces, ces écotones, entre la terre et l'air, la ville et ses abords. Le sol peut être relégué à une fonction de filtre commode [...] Il garde la mémoire de la ville et des hommes qui l'ont faite, mémoire organique des dépotoirs et des sépultures, chimique de la Révolution industrielle, mécanique des charges qu'il a portées, mémoire révélée à l'occasion des éventrations fréquentes qui mettent au jour une terre infecte, souillée par les activités industrielles [...] » (Barles, 1999, p. 335). D'après J.M. Roy ${ }^{14}$, « on ne se rend pas compte du risque lié au plomb ou au cadmium (etc.) que peuvent contenir les boues [...] Avant 1980-90, on ne se soucie absolument pas de la pollution des sols dans le grand public, on n'en parlait absolument pas, la terre avait quelque chose de naturel ». Il faudrait des études historiques plus approfondies pour décrypter les raisons de cette pratique d'invisibilisation des pollutions qui est sans nul doute multi-factorielle; tout au moins pouvons-nous envisager quelques pistes de réflexion. Il faut souligner tout d'abord des raisons techniques car les premiers appareils de spectroscopie d'absorption atomique permettant de doser les ETM ne sont apparus dans les laboratoires qu'au début des années 1970, même si d'autres techniques existaient, le saturnisme étant connu depuis longtemps ${ }^{15}$. A cela s'ajoutent des rapports de force au XIX siècle entre la ville de Paris et sa périphérie (Barles, op.cit.). En effet, il n'est pas inutile de rappeler que le mouvement hygiéniste s'est réalisé certes à Paris, mais au détriment de la proche banlieue qui a été polluée par le transfert des déchets urbains parisiens, soulevant par la même des questions plus actuelles de justice environnementale (Larrère, 2009 ; Blanchon et al., 2009). C'est précisément cet héritage du sol via les transferts de terres contaminées que met en exergue notre étude de cas.

Lorsqu'en 1860, Paris absorbe ses communes suburbaines, les maraîchers quittent la capitale pour investir de gros lotissements comme Stains, Créteil, ou encore la nouvelle ville de Montrouge, amputée d'une partie de son territoire (figure 2). L'activité maraîchère n'a de cesse de péricliter au fil du XXe siècle et lorsque le département de la Seine est supprimé en 1968 au profit des quatre nouveaux départements (Paris, Hauts-deSeine, Val-de-Marne et Seine-Saint-Denis), les nouvelles préfectures se sont constituées là où il y avait de l'espace vacant, à savoir sur les terrains maraîchers (figure 2); ce qui est particulièrement vrai pour les préfectures de Bobigny (Seine-Saint-Denis) et de Créteil (Val-de-Marne). En outre, même si ce phénomène a débuté à l'aube de la Première Guerre mondiale, beaucoup de maraîchers ont arrêté leur exploitation entre 1940 et 1960. C'est précisément cette terre vendue qui a servi à façonner les espaces verts parisiens comme le précise J.M. Roy ${ }^{16}$ " Quand on a créé les préfectures de Bobigny et de Créteil, toute la terre végétale a été vendue à la ville de Paris pour ses espaces verts. Tous les maraîchers le savaient. Un maraîcher pouvait gagner sa vie en vendant de la terre ou du terreau [...] C'était dans la vulgate maraîchère, tous les maraîchers me l'ont dit, le plus gros client en terre sont les EV de Paris ${ }^{17}$.

Si on se place dans cette perspective historique, on constate que cette ingénierie d'aménagement urbain a conçu l'assainissement de la ville en recyclant les déchets urbains sur les espaces agricoles (ou en sous-sol). Pourtant, l'impact de ces pratiques en 
termes de contamination des sols reste peu abordé lorsqu'on parle d'économie circulaire, sauf cas extrême comme à Achères et Pierrelaye (Baize et al., 2009).

Figure 2 : Evolution des maraîchers et de leur répartition spatiale entre 1859 et 1959

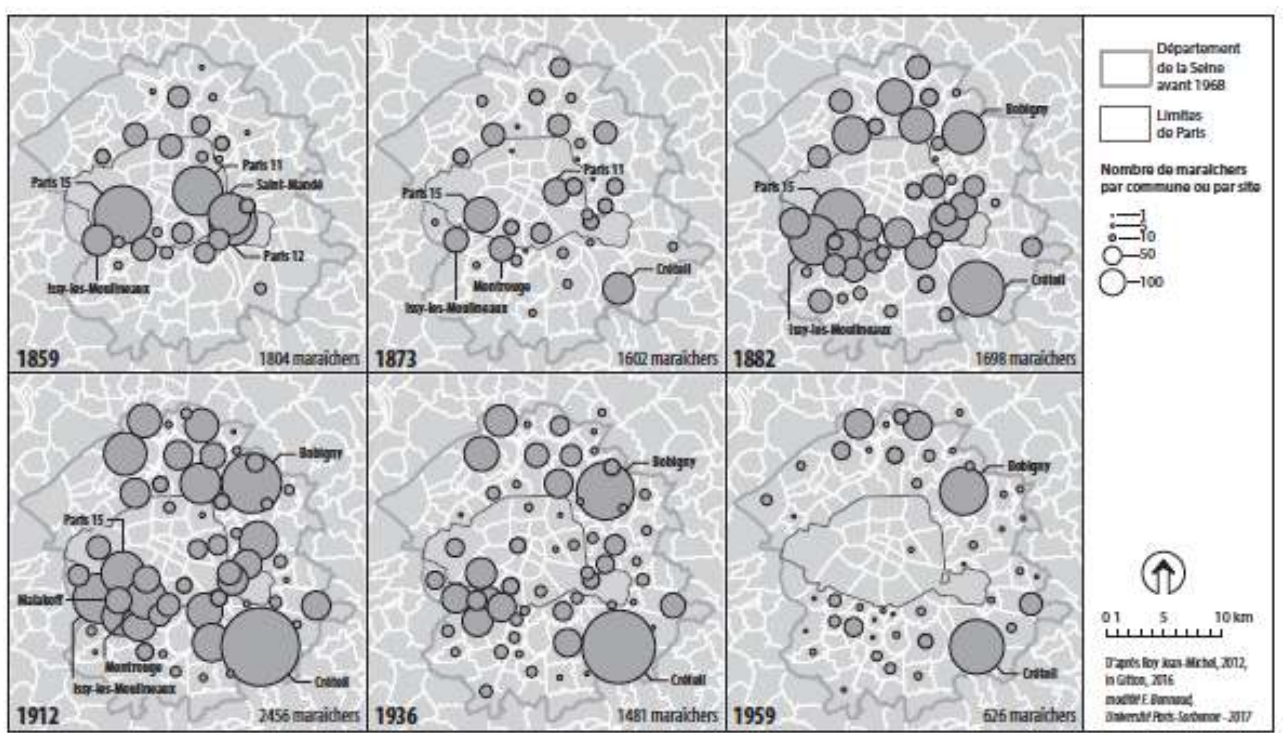

D’après Roy Jean-Michel, 2012, in Gitton, op. cit

19 Ainsi, l'analyse historique des transferts de «terre végétale » semble être une piste intéressante quant à l'explication des polluants présents dans certains EV parisiens : les terres d'apport pourraient avoir été préalablement contaminées. Au vu des résultats des contaminations en ETM obtenus lors de campagnes d'analyses des terres des EV, on avance alors l'hypothèse que de telles concentrations en ETM sont directement en lien avec la qualité des terres d'apport au cours des différentes périodes d'aménagement. La confrontation avec des mesures prises sur le terrain dans le cadre de ce travail permettra (ou non) d'étayer cette piste (Canolle, op. cit.; Gitton, op. cit.). 
Figure 3 : Essai de synthèse : frise des sources probables de contamination en ETM pour les EV parisiens depuis 1800

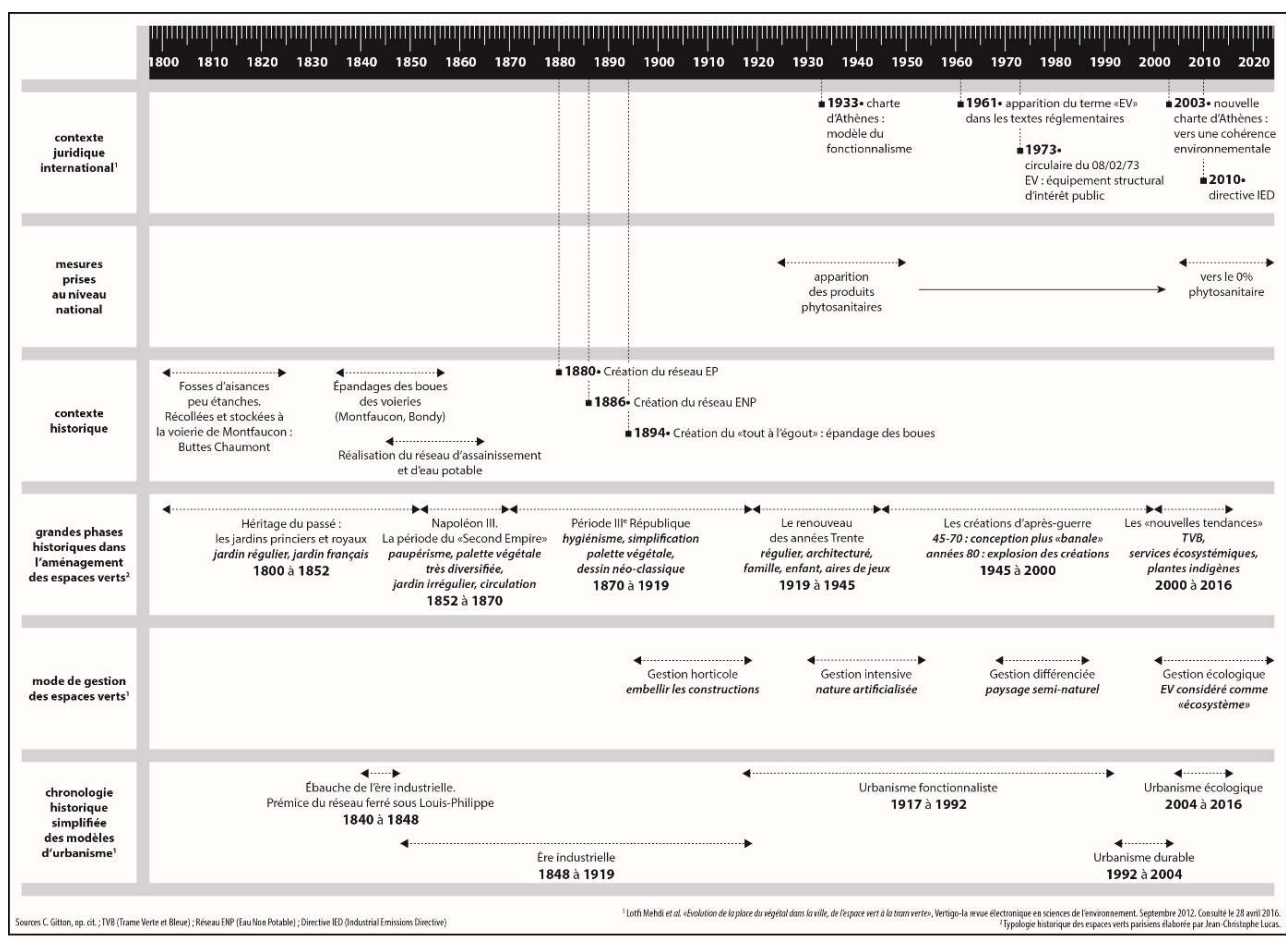

Essai de synthèse : frise des sources probables de contamination en ETM pour les EV parisiens depuis 1800.

Sources : Gitton, op. cit. ; TVB (Trame Verte et Bleue) ; Réseau ENP (Eau Non Potable) ; Directive IED (Industrial Emissions Directive).

\section{Hypothèse sur l'origine des terres grâce aux données historiques, pédologiques et agronomiques}

\section{Méthodologie.}

\section{Le choix des sites}

La démarche a reposé sur la caractérisation de sols d'EV pour refléter la diversité des situations, à savoir les différentes phases d'aménagement et la diversité de leur superficie. La base de données de la Direction des Espaces Verts et de l'Environnement, référençant tous les espaces verts de Paris, a été utilisée de façon à orienter le choix des sites pour obtenir une représentativité historique, géographique, surfacique, etc. La synthèse historique schématisée dans la figure 3 a été utile pour établir cette sélection. Sur les 102 sites sélectionnés, les données existantes de 49 sites ont été compilées à partir de la base de données du laboratoire d'agronomie de la ville de Paris (Canolle, op. cit.) qui ont ensuite été complétées par une campagne de sondages sur 53 sites (Figure 4, Canolle, Gitton 2016). 
Figure 4 : Cartographie des espaces verts échantillonnés dans le cadre de l'étude sur la qualité des sols des espaces verts parisiens

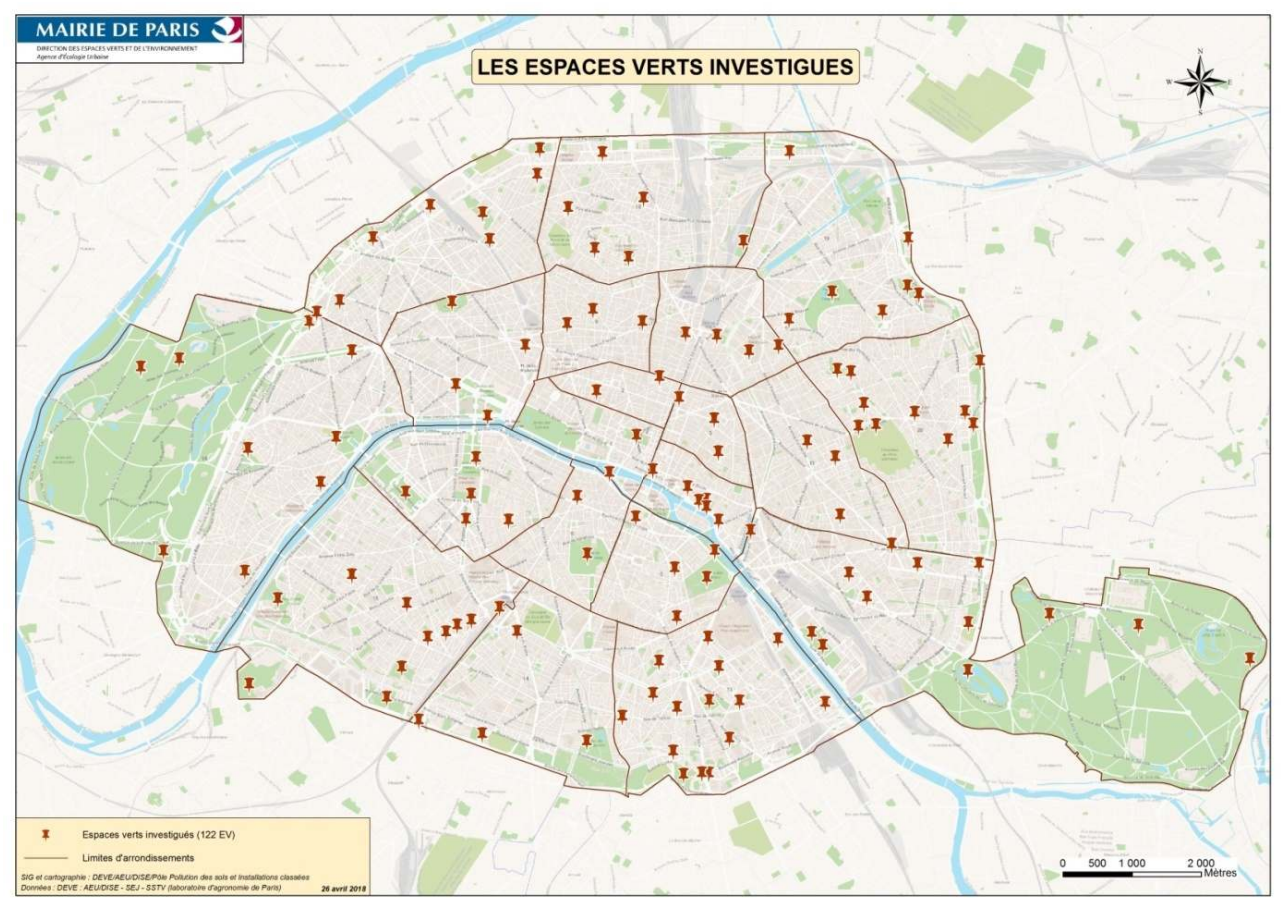

Sources : E. Canolle et C Gitton, op. cit.

Figure 5 : Répartition du nombre d'EV étudiés selon la typologie établie pour l'étude

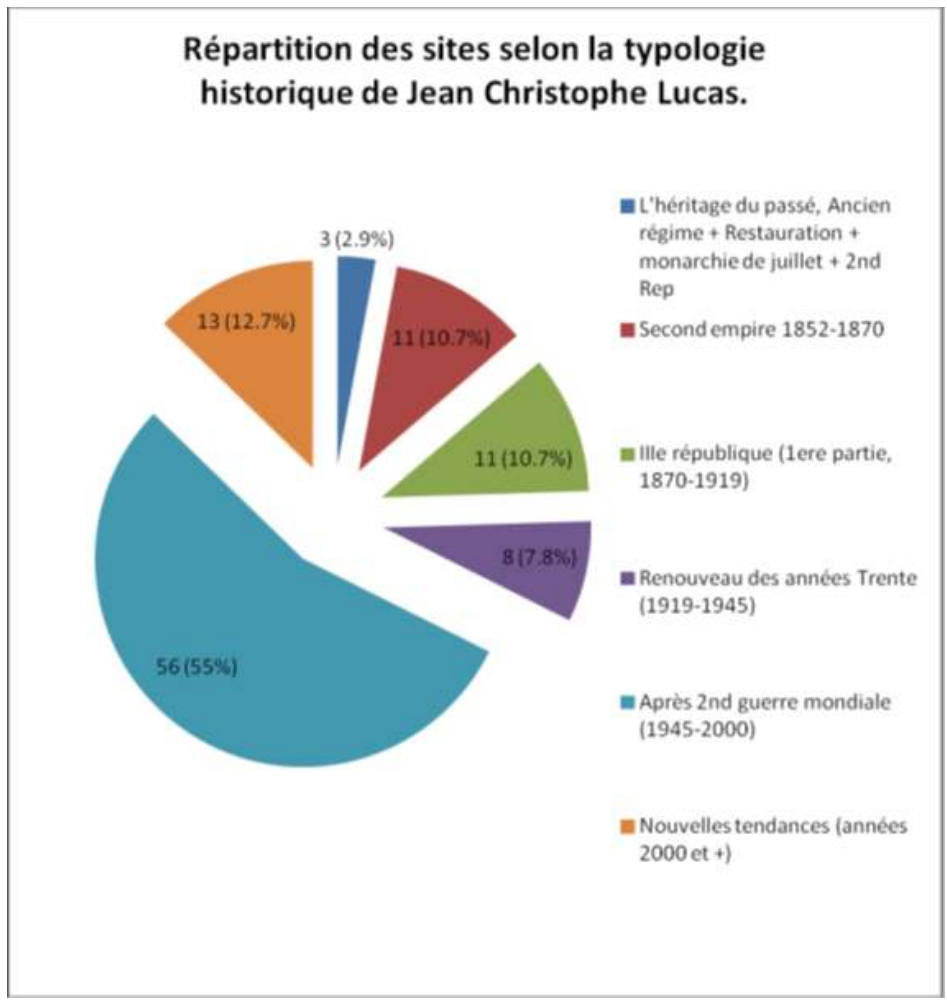

Source : C. Gitton, op. cit 
Bien que l'on observe une prédominance des EV datant de la période d'après-guerre (55\% ), la sélection des sites par période est représentative de la situation à l'échelle de Paris car près des deux tiers de ces surfaces ont été analysées (à savoir 333 ha sur près de 500 ha).

\section{Méthodologie des mesures}

Tous les prélèvements de sol sont des composites de 5 prélèvements unitaires sur $1 \mathrm{~m}^{2}$ géo-localisés. Le nombre de prélèvements par $\mathrm{EV}$ a été défini pour être représentatif de la qualité des sols de l'EV. Une étude de cette représentativité a été réalisée sur 9 sites sur la base de mesures par fluorescence $\mathrm{X}$. Les prélèvements de sols ont été réalisés à l'aide d'une tarière à main à 2 profondeurs différentes $(0-5 \mathrm{~cm}$ et $5-30 \mathrm{~cm})$ pour appréhender une éventuelle hétérogénéité verticale des paramètres mesurés. Les résultats des analyses n'ayant pas montré de différence significative entre les 2 profondeurs, les résultats de la profondeur 0-5 cm, ont été exploités pour cette étude.

Un total de 285 prélèvements composites de terre a ainsi été analysé au laboratoire d'agronomie de la ville de Paris. Les analyses concernent les paramètres pédologiques, agronomiques et environnementaux des sols des $\mathrm{EV}: \mathrm{pH}$, résistivité, concentration en carbone organique, et azote permettant le calcul du rapport $\mathrm{C} / \mathrm{N}$, proportion en sables, teneurs en calcaire total, phosphore total, et concentrations en éléments trace métalliques (arsenic, cadmium, chrome, cuivre, mercure, nickel, plomb, sélénium et zinc).

\section{Résultats.}

\section{Origine et qualité agronomique des terres végétales utilisées pour la création ou les aménagements des EV.}

\section{Les paramètres pédologiques et agronomiques des sols des EV}

24 Les paramètres suivants ont été déterminés systématiquement sur l'ensemble des prélèvements: $\mathrm{pH}$, résistivité, carbone organique, et azote permettant le calcul du rapport $\mathrm{C} / \mathrm{N}$, proportion en sables, teneurs en calcaire total, phosphore total et concentrations en éléments trace (arsenic, cadmium, chrome, cuivre, mercure, nickel, plomb, sélénium et zinc). Ces paramètres physico-chimiques sont utilisés comme des indicateurs des usages passés des terres, jouant par là même un rôle de mise en visibilité de leur contamination potentielle.

Dans le cadre de ce numéro thématique, le choix a été fait de ne présenter que quelques paramètres :

26 - plomb, phosphore et matière organique, car connus comme étant représentatifs des sols urbains (Joimel et al., 2016)

27 - teneur en sables et calcaire, car permettant de discriminer la nature des sols de la région parisienne.

L'exploitation de ces paramètres est présentée de manière descriptive au regard des quatre grandes périodes d'aménagement différenciées (figures 6 à 9). Précisons qu'une analyse statistique approfondie fera l'objet d'un article à venir spécifique (Nold et al., en préparation). 
Figure 6 : Pourcentage d'EV en fonction des teneurs en sables (\%) dans les sols selon les périodes d'aménagement

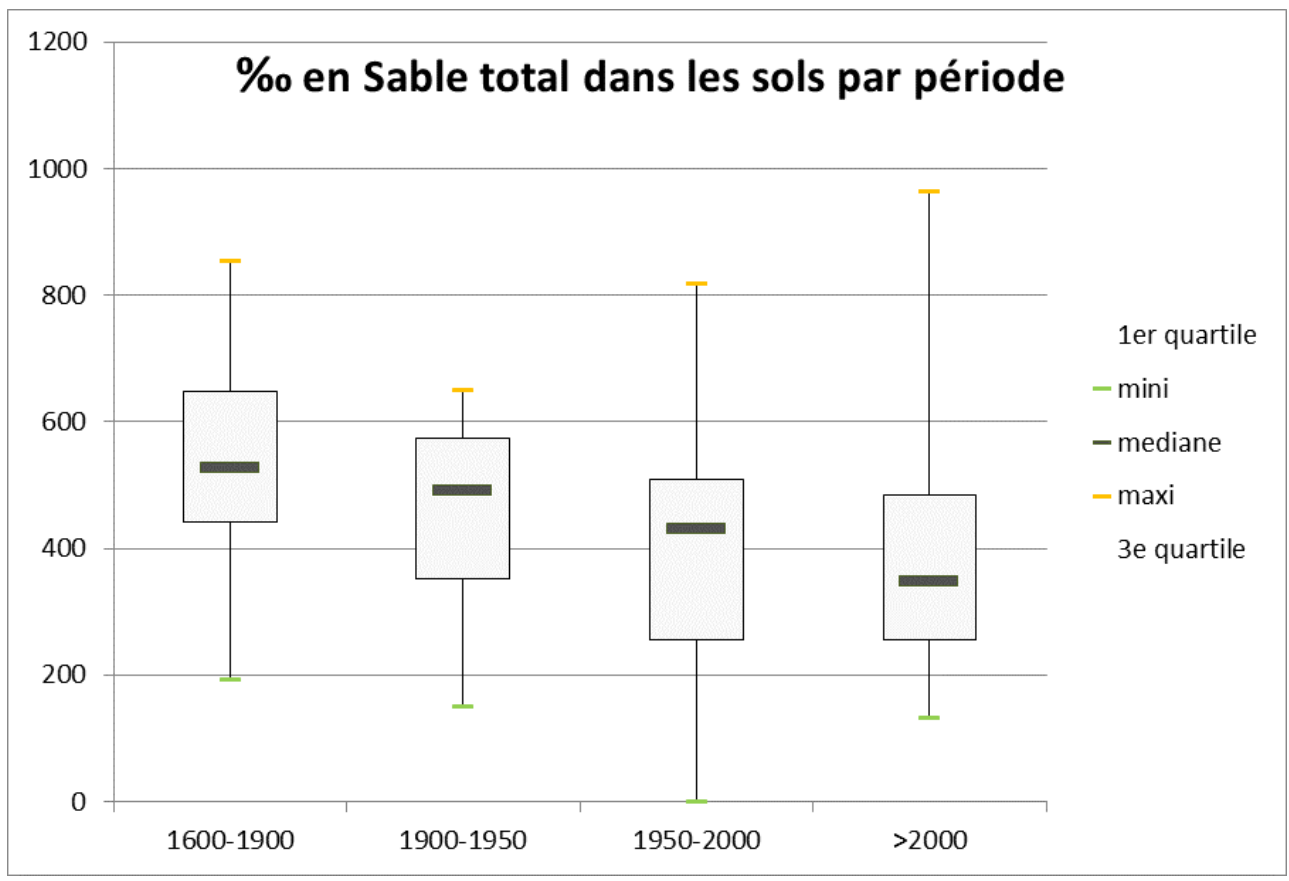

Source : Gitton, op. cit.

Figure 7 : Répartition des pourcentages de calcaire total dans les sols selon les périodes d'aménagement

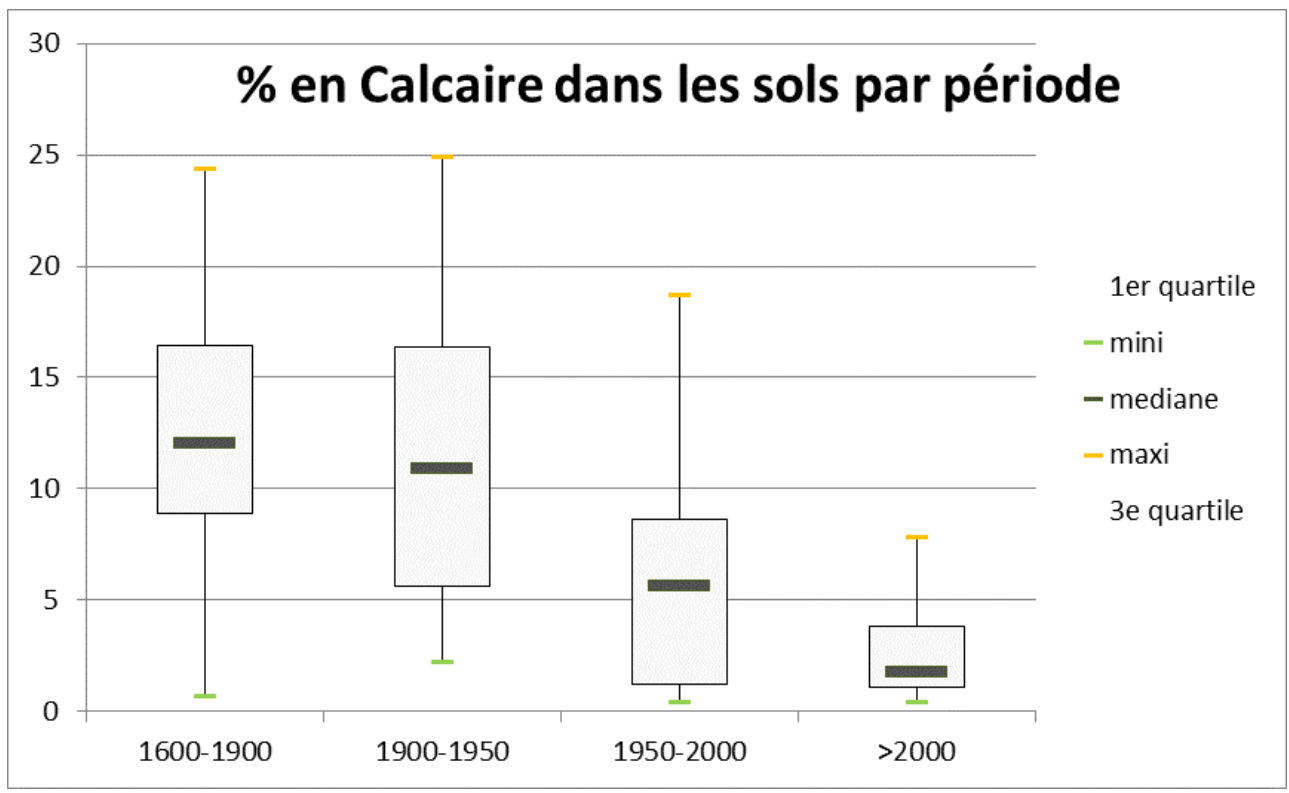

Source : Gitton, op. cit. 
Figure 8 : Pourcentage d'EV en fonction des teneurs en matière organique (\%) dans les sols selon les périodes d'aménagement

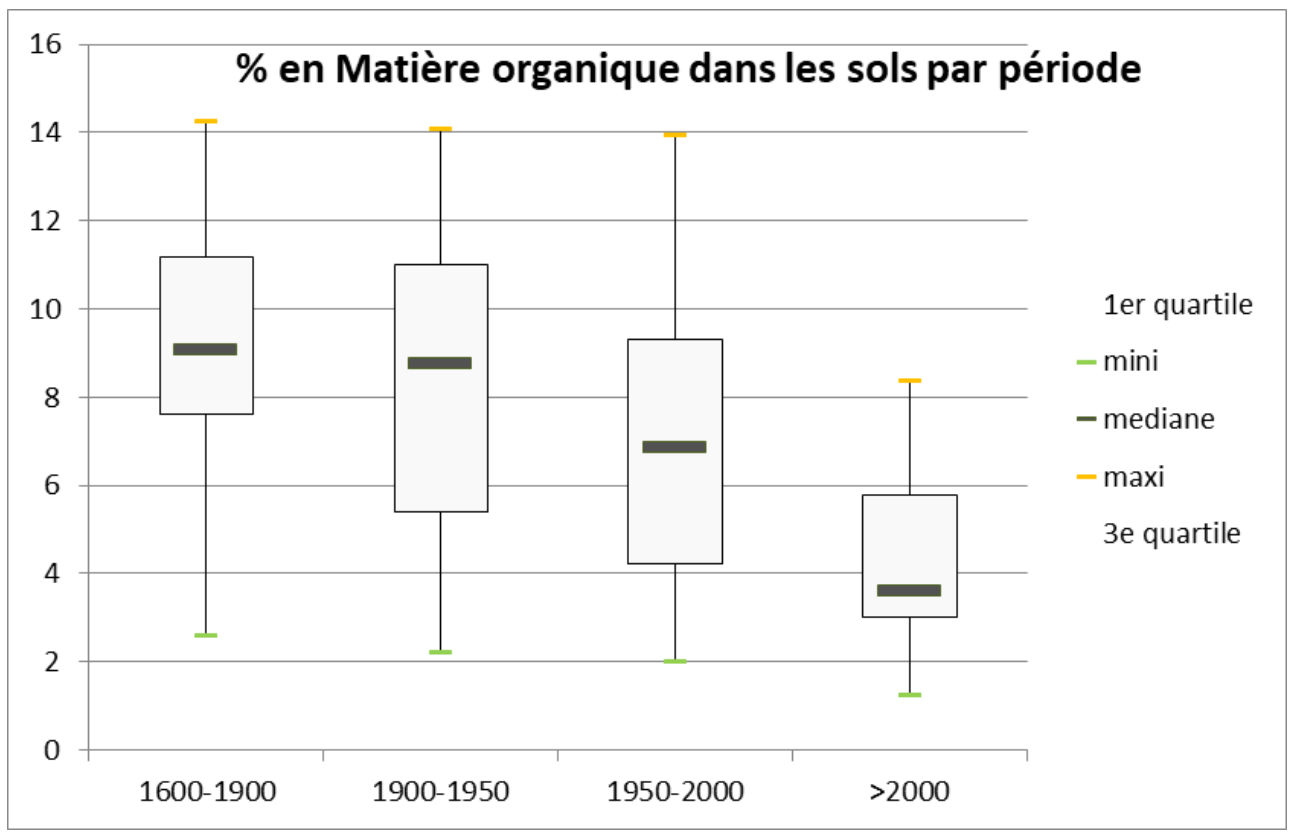

Source : Gitton, op. cit.

Figure 9 : Pourcentage d'EV en fonction des teneurs en phosphore assimilable (\%) dans les sols selon les périodes d'aménagement

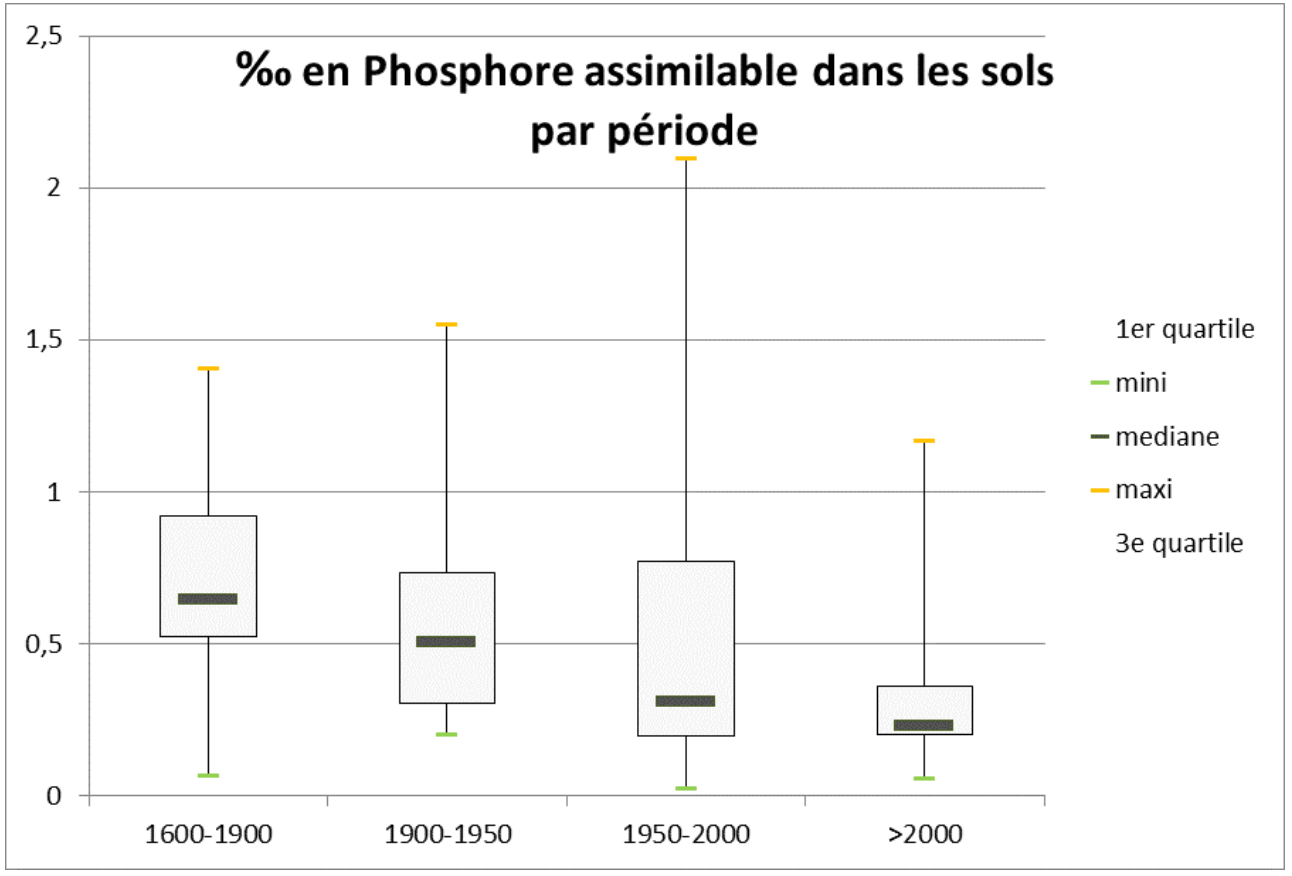

Source : Gitton, op. cit.

L'analyse de ces quatre paramètres permet de faire l'hypothèse de deux origines différentes concernant la provenance des terres d'apport en lien avec la période d'aménagement. En effet, les sols des EV aménagés avant 1950 présentent une teinte foncée cohérente avec la détermination des teneurs élevées en matière organique, ainsi 
qu'une forte teneur en sables ${ }^{18}$ (texture limono-sableuse). Elles contiennent aussi des taux élevés en phosphore assimilable et en calcaire. Cette terre pourrait provenir d'une terre maraîchère.

Les sols d'EV aménagés postérieurement à 1950 sont de teinte plus claire, en cohérence avec le fait qu'ils sont moins riches en matière organique. La texture n'est plus dominée par les sables, elle est argilo-limoneuse. Les teneurs en matière organique sont plus faibles, le calcaire total est quasiment absent $(<3 \%)$. Les paramètres agronomiques correspondent aux terres des plaines de grande culture de l'ile de France (sols bruns lessivés ou «limons des plateaux » de Beauce et de Brie). Comme le montre la figure 10, les terres noirâtres (à gauche) sont plus riches en matières organiques que les terres brunâtres (à droite). Les premières, plus anciennes, sont associées à une origine maraîchère.

Figure 10 : « L'origine de la terre des espaces verts parisiens »

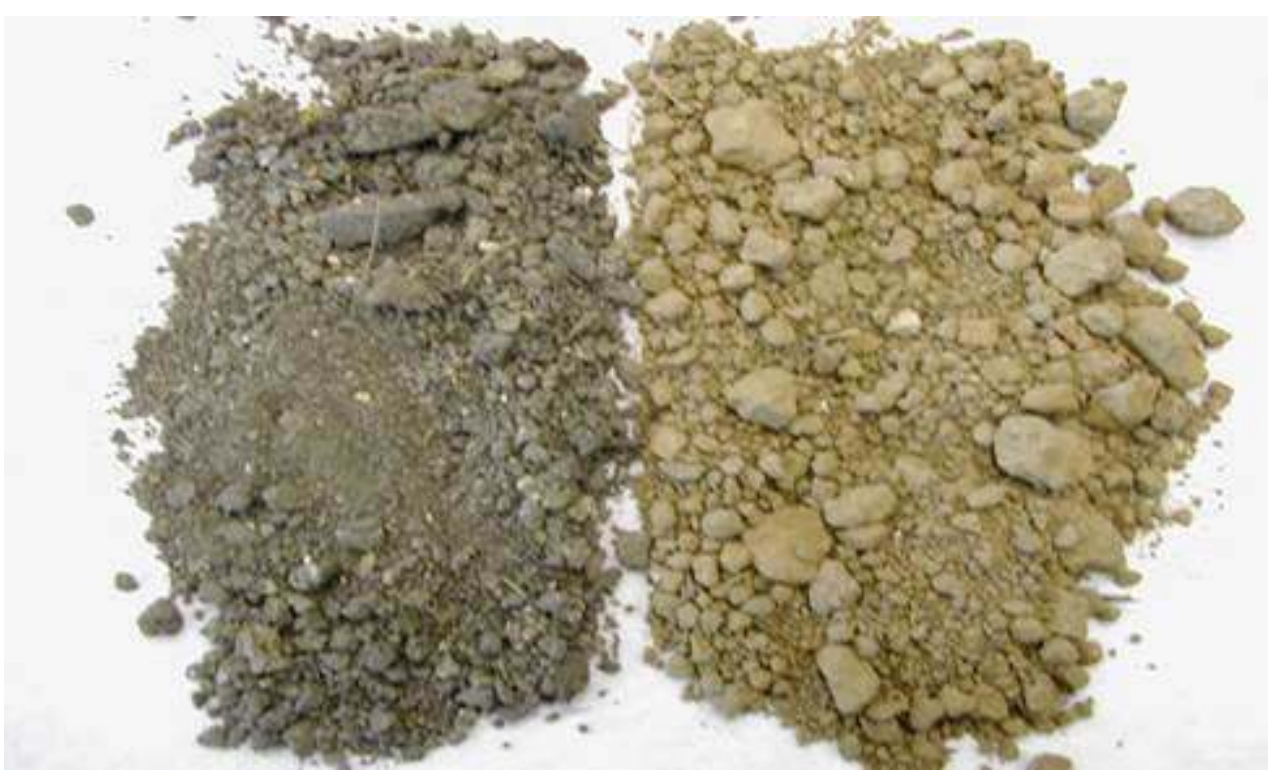

Source : F. Nold, Cours d'agronomie urbaine, Licence Professionnelle ECOPUR.

31 Ces résultats semblent confirmer l'hypothèse que les espaces verts parisiens ont été réalisés avec des apports de terre provenant de terrains maraîchers jusqu'aux alentours de 1950. Au-delà de 1950, les terres utilisées correspondent davantage à des sols de grande culture.

\section{Degré de contamination en ETM des sols des EV}

La concentration en plomb dans les sols est un indicateur reconnu de la pression anthropique sur le milieu (Joimel et al., 2016). Ainsi, les sols (péri)urbains présentent classiquement des concentrations en plomb sensiblement supérieures à celles de sols agricoles. Bien que la proximité de certains sites industriels ou d'axes de circulation (principalement avant les années 2000) a pu accroître notablement les concentrations en plomb, les concentrations les plus élevées retrouvées dans les sols ne peuvent s'expliquer par la seule pollution introduite par retombées atmosphériques au regard des flux observés et d'absence de corrélation entre la localisation des EV et les axes de circulation. L'hypothèse de la contamination repose donc sur le fait qu'une des sources les plus 
importantes en termes de quantité en polluants reste l'utilisation de certaines pratiques agronomiques comme l'épandage d'eaux résiduaires ou gadoues urbaines. Comme signalé plus haut, ces gadoues ou boues de villes constituées des balayures des rues, des halles et des marchés étaient très employées par les cultures maraîchères à Paris (Larbaletrier, 1898).

L'examen des concentrations en plomb dans les sols montre clairement que celles-ci sont fonction de la période d'aménagement ${ }^{19}$ (figure 11). Ainsi, pour les aménagements réalisés avant 1950 sont observés les plus importants pourcentages d'EV ayant des concentrations les plus élevées en plomb dans les sols. Pour la période après 2000, les pourcentages les plus élevés concernent les EV ayant des concentrations les plus faibles en plomb. La période intermédiaire (1950-2000) montre une contamination plus hétérogène. Ceci pourrait s'expliquer par des mélanges de terres ${ }^{20}$ et une contamination par des terres plus anciennes ou des dépôts extérieurs. La présence d'ETM dans les terres importées récemment peut également s'expliquer par une défaillance $\mathrm{du}$ contrôle d'approvisionnement. A défaut d'une surveillance attentive, il n'est pas interdit de penser que des terres évacuées d'un jardin en rénovation trouvent une nouvelle vie dans la création ou la rénovation d'un espace vert voisin. C'est l'une des raisons pour laquelle on rencontre quelques terres contaminées dans des EV postérieurs à 2000 .

Figure 11 : Pourcentage d'EV par période d'aménagement en fonction de la concentration en plomb $(\mathrm{mg} / \mathrm{kg})$

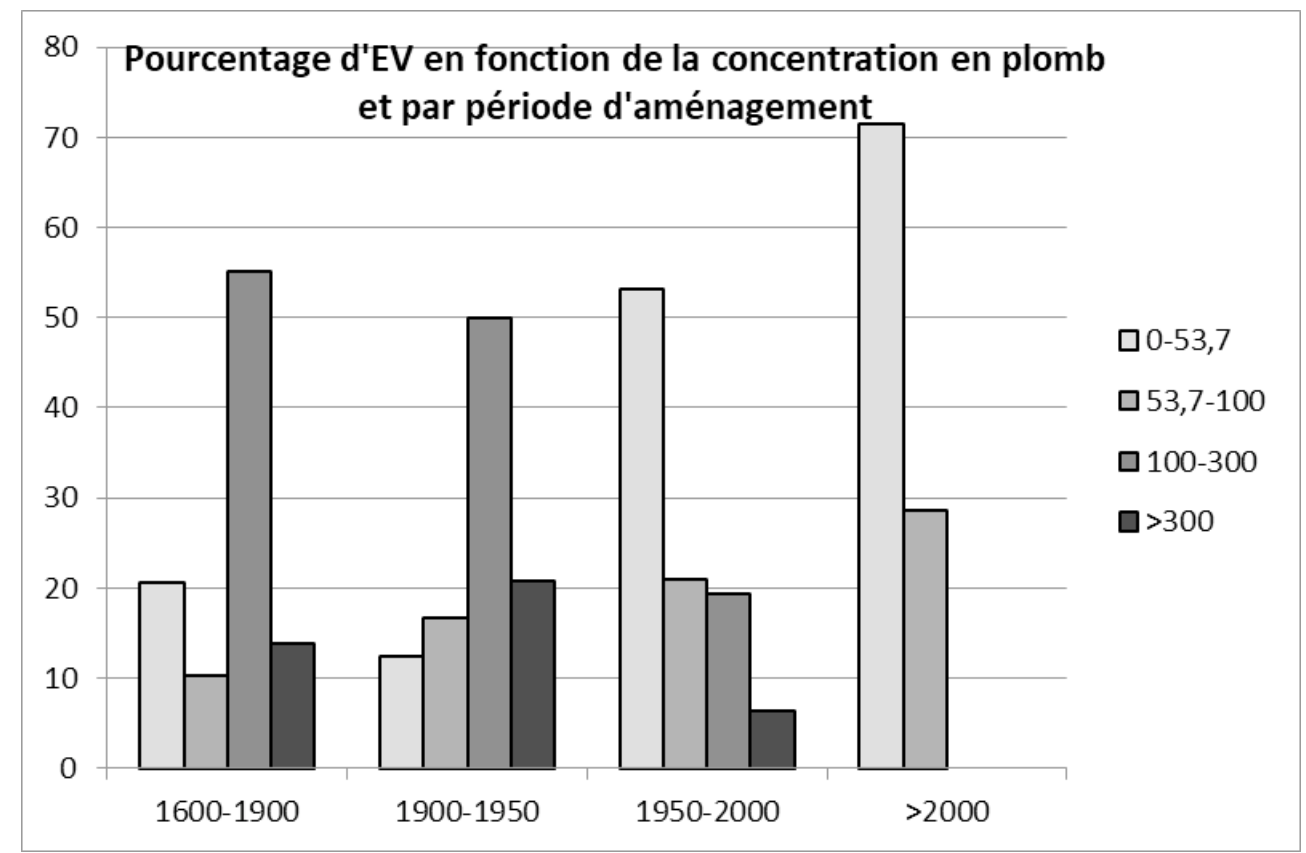

Source : Gitton, op. cit.

Comme nous l'avons déjà montré, certains paramètres agronomiques sont caractéristiques de l'origine de la terre comme le pourcentage de calcaire élevé pour les terres maraîchères. La figure 12 montre la corrélation qui existe entre ce pourcentage de calcaire par exemple et la concentration en plomb dans les EV. 
Figure 12 : Corrélation entre la concentration en Plomb $(\mathrm{mg} / \mathrm{kg})$ et le pourcentage en calcaire total dans les EV parisiens

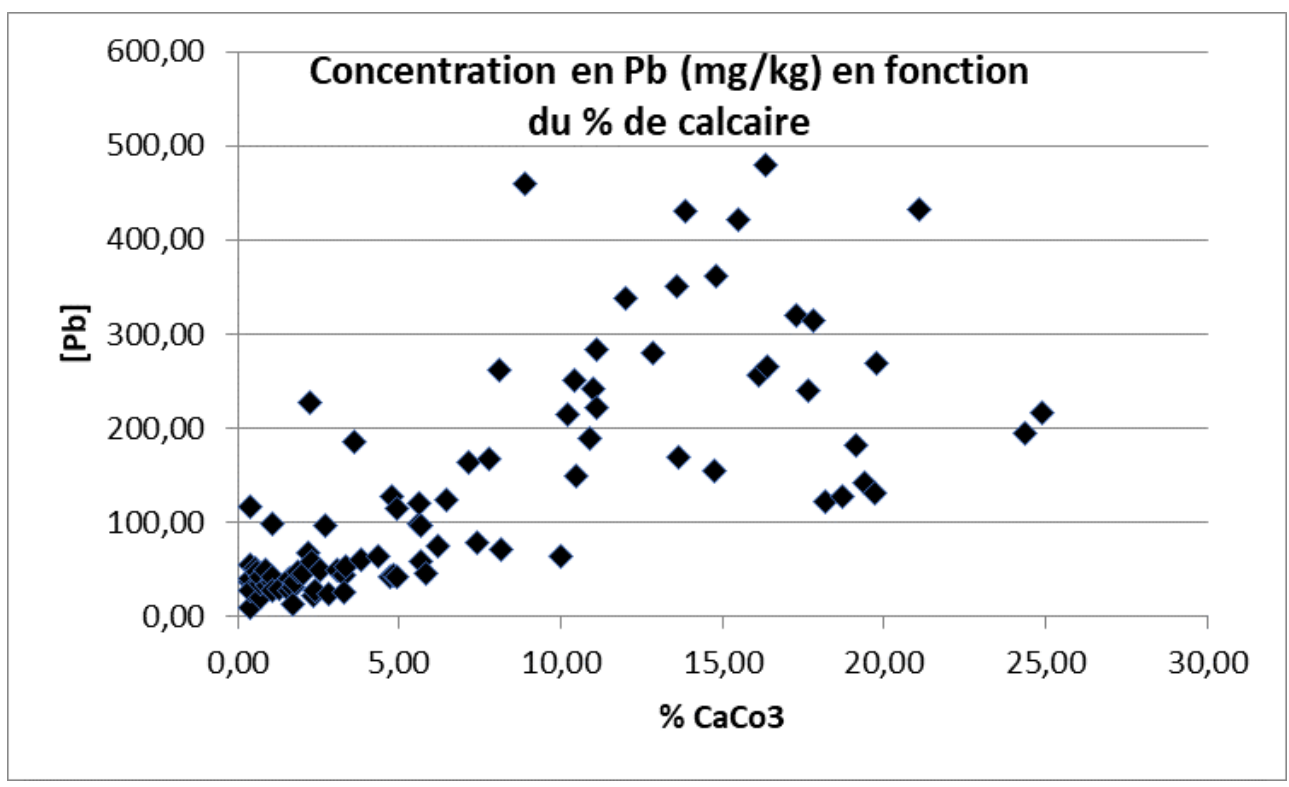

Source : Gitton, op. cit.

Ainsi, la contamination en plomb, et en autres éléments, constatée dans les sols des EV aménagés avant 1950, est ici expliquée par l'utilisation de terres maraîchères apportées de la périphérie parisienne lors de leur création ou de leur aménagement et in fine, par les anciens épandages d'eaux usées urbaines. En ce sens, les contaminants ont réintégré l'espace urbain après avoir été évacués avec les eaux usées dans un but d'assainissement.

\section{Débat et conclusion}

$\mathrm{Au}$ terme de ce travail visant à analyser l'aménagement des espaces verts parisiens via l'apport de «terres végétales », il semble utile de réinterroger les principes de l'économie circulaire appliqués à notre cas d'étude. Cette dynamique qui théoriquement crée une boucle vertueuse, peut-elle aussi dans certains cas, engendrer une boucle à effets négatifs? Quels enseignements en tirer quant à la dialectique visibilité/invisibilité des risques associés aux sols (péri)urbains pressentis pour l'aménagement des territoires récréatifs et alimentaires urbains?

La thématique de la pollution des sols n'apparaît presque pas dans le champ du métabolisme urbain et de l'écologie urbaine, si ce n'est dans des travaux reliant l'exposition à un environnement pollué avec la classe sociale des habitants (Cousins and Newell, 2015), ce qui représente une autre forme d'injustice sociale et environnementale. Sabine Barles (2002), dans sa revue de littérature sur les liens entre écologie et métabolisme urbain, cite seulement deux travaux portants sur les stocks de polluants, l'un sur la pollution au mercure à Stockholm (Jonsson, Svidén 1999), et l'un sur la pollution au plomb en France (Lestel, 2002) et conclut (p. 148-149): «D'autres pistes de réflexion auraient pu être abordées sur les rapports qu'entretiennent la ville et son environnement et leur évolution. Force est de constater que nous savons encore peu de ceux-ci, qu'il s'agisse du passé ou du présent ». L'apport de notre étude se situe au niveau de cette interaction en 
croisant une investigation sur la qualité des terres d'apport issues d'anciennes terres maraîchères et la pertinence de leur réutilisation à l'appui d'une analyse historique sur l'aménagement des espaces verts parisiens.

Si l'on résume les résultats obtenus dans cette étude exploratoire, on se rend compte de l'effet négatif et à rebours d'une pratique à l'origine peu vertueuse : l'assainissement des différents quartiers parisiens s'est effectué au XIXe siècle en particulier en évacuant les «boues » urbaines non traitées, potentiellement chargées en polluants dont des métaux lourds (Barles, 1999) et dans des proportions difficilement quantifiables, vers des communes périphériques maraîchères (Dron, 2009). Celles-ci ont approvisionné en terres végétales les quartiers parisiens dont étaient initialement originaires les boues exportées ...

Par la mise en visibilité qu'ils autorisent, les présents travaux, dont seules les données relatives au plomb ont été présentées ici, permettent une première évaluation sérieuse du degré de contamination en éléments traces et des paramètres agronomiques des espaces verts parisiens.

Les corrélations établies entre les paramètres agronomiques et les concentrations en ETM dans les sols, reliées à l'historique des sites, ont permis de constater que les aménagements antérieurs à 1950 avaient vraisemblablement reçu un apport de terre maraîchère sur laquelle avait été déversée des eaux usées brutes ou des boues. Lors de leur disparition ou de leur déplacement sous la pression de l'urbanisation, ces terres maraîchères ont été utilisées pour l'aménagement des EV parisiens. Elles possèdent des caractéristiques particulières confirmées par les mesures, engendrées par les importants épandages qu'elles ont reçus, et qui ont certainement généré la contamination observée. Il est aussi intéressant de souligner que les cahiers des charges en usage à la Ville de Paris, y compris les plus anciens, mentionnent « l'interdiction des terres maraîchères ", ce qu'il était quasiment impossible de vérifier avant le recours aux analyses. La suspicion concernant la qualité de ces terres reposait auparavant sur un savoir tacite des services techniques de la ville, crainte que nos analyses ont confirmée. Celles-ci contribuent à la mise en visibilité des risques potentiels associés à ces terres maraîchères car il existe désormais une capitalisation des connaissances et une traçabilité des polluants associés. Cette dynamique cognitive contribue à la mise à l'agenda de la question de la contamination des sols urbains.

41 L'examen réalisé sur la typologie historique met en exergue l'importance de l'histoire de l'aménagement paysager sur la contamination en éléments traces métalliques des espaces verts de la capitale. Certaines périodes dégagées dans l'étude sont beaucoup plus marquées par l'impact de la pollution que d'autres. Ainsi, les périodes les plus anciennes sont celles auxquelles correspondent les plus fortes proportions de sols pollués. A la faveur du facteur historique, une source prépondérante de contamination a été identifiée : l'apport en "terre végétale ». En effet, les espaces verts parisiens en ont toujours reçu lors de leur aménagement. C'est ainsi que ces terres, selon leur origine, ont pu être contaminées par des pratiques horticoles (maraîchères ou légumières) néfastes $e x$ situ (épandages ou amendements). Si bien que l'étude a mis en évidence une phase-clef, celle des années 1950, comme étant la période de changement de composition et de qualité des « terres végétales » d'apport, confirmant ainsi notre hypothèse initiale.

Après 1950, les terres d'apport sont issues de zones agricoles, moins impactées par les épandages et assujetties à des prescriptions et des contrôles de qualité à partir des années 1980 , tout d'abord sur les paramètres agronomiques puis, plus récemment, sur les 
concentrations en ETM des sols. Toutefois pour être plus exhaustif et précis sur les périodes les plus récentes, il ne faut pas négliger le développement et l'accroissement de l'usage des pesticides qui ont été épandus en zones rurales de grandes cultures et qui, après transfert vers le sol, pourraient, dans le cas des substances les plus rémanentes, à leur tour, contaminer la ville; il ne faudrait pas non plus négliger les polluants dits "émergents", perturbateurs endocriniens ou d'origine médicamenteuse comme les antibiotiques qui peuvent se retrouver dans les boues de station d'épuration car difficiles à éliminer.

Alors que la mairie de Paris développe la végétalisation de la ville, favorise l'essor des jardins partagés au sein de ses espaces verts et accompagne des projets d'agriculture dite urbaine, la vigilance sur le contrôle des terres d'apports apparaît alors cruciale ${ }^{21}$. Bien qu'on ne puisse exclure une contribution provenant de la pollution atmosphérique (Foti, 2017) ou encore des composts urbains élaborés à partir de déchets de la ville, il apparaît que la contamination des terres végétales d'apport des EV soit due en premier lieu à l'utilisation d'eaux usées brutes ou de boues sur les terres maraîchères.

Les contaminations potentielles et avérées en ETM, associées à la réimportation des terres souillées par les déchets de la ville préalablement rejetés à sa périphérie, invitent à aborder les principes de l'économie circulaire avec quelques précautions. "Nourrir la ville par la ville » est donc plus complexe qu'il n'y paraît. Ces résultats invitent également à envisager de façon plus relative les concepts de "villes vivrières", d'" agrarisation vertueuse de la ville » ou de « sol nourricier », qui, pertinents dans certains cas, peuvent néanmoins faire l'objet d'un certain nombre de réserves (Ernwein, Salomon-Calvin, 2014). La démarche de production et de consommation locale doit être conçue en tenant compte du substrat, c'est-à-dire du sol souvent considéré à tort comme un simple support inerte, sans oublier l'environnement alentour (Canavese et al. op.cit.). Paradoxalement, dans un contexte où le développement de "l'agriculture urbaine " est mis en avant dans les projets de ville durable, ne doit-on pas envisager au contraire de rendre compatible les usages urbains (récréatifs ou de production alimentaire) en fonction de la qualité (agronomique mais aussi physico-chimique) de ces sols ${ }^{22}$ ? D'ailleurs, le verdissement de la ville et au-delà de façon plus complexe l'entretien d'une biodiversité urbaine, doiventils être associés à des productions végétales comestibles alimentant des circuits courts?

Dans son principe général, l'économie circulaire peut contribuer à une invisibilisation de la question des terres polluées si on ne prend pas la peine de questionner la qualité du sol (péri)-urbain en lien avec son histoire. En inversant le regard, nous avons tenté de montrer comment certaines pratiques ont pu impacter la qualité de ces terres utilisées pour le jardinage et s'inscrire, dans ce dernier cas, de façon inappropriée dans des boucles alimentaires. Cela permet également de faire le constat qu'il peut y avoir des contreparties négatives non envisagées initialement dans les projets d'aménagement urbain. D'hier à aujourd'hui, il s'agit finalement de se détacher d'une vision utilitariste du sol qui passe, non sans paradoxe, d'une vision du sol "épurateur de déchets " $^{23}$ à celui de support productif, comme si le sol ne gardait pas en mémoire les traces des usages passés. Emerge ainsi l'idée d'un sol comme un « actant » (Latour, 2006) à part entière ayant sa propre dynamique, pas toujours conforme à l'usage qu'on voudrait lui attribuer a priori.

En guise d'ouverture et à titre d'hypothèse, souligner les limites de l'économie circulaire peut permettre d'engager une démarche de résilience, si l'on comprend ceci dans le sens où elle peut permettre une réduction des impacts environnementaux négatifs et donc une augmentation de la réutilisation de terres locales non polluées. En effet, l'économie 
circulaire n'est résiliente - i.e. elle permet d'améliorer la capacité du système à rester stable et à s'affranchir des changements de son environnement (Holling, 1973) - que si elle évacue les polluants délétères de son cycle et si elle parvient à diminuer sa consommation de matières premières provenant de l'extérieur. Notre article peut favoriser une prise de conscience permettant l'émergence d'une économie circulaire plus "propre", et ainsi contribuer à une plus grande résilience du système. Développer et diffuser les connaissances sur la question de la qualité du sol (péri)urbain ${ }^{24}$ est un domaine sans nul doute à investir pour mieux produire «la ville sur la ville » sans négliger toutes les autres fonctions que peuvent rendre les sols urbains lorsqu'ils ne sont pas imperméabilisés: stockage du carbone, biodiversité, régulation thermique et hydrique par infiltration et rétention des eaux de pluie. Finalement, il importe de mieux connaître les sols urbains et péri-urbains sans négliger leur histoire pour créer in fine une boucle plus vertueuse.

\section{BIBLIOGRAPHIE}

ADEME, 2018, Economie circulaire, en ligne http://www.ademe.fr/expertises/economie-circulaire.

AUGUSTSSON, A., SÖRME, L., KARLSSON, A., \& AMNEKLEV, J. 2017, Persistent Hazardous Waste and the Quest Toward a Circular Economy: The Example of Arsenic in Chromated Copper Arsenate-Treated Wood, Journal of Industrial Ecology, vol 21, n³, p. 689-699.

BAIZE D., HELBEOC' H A., BLONDEL T., CHAUSSOD R., DELMAS A-B., DEROUBAIX J-F., DE SORAS O., GASPERI J-M., JAUZEIN M., LAMY I., VAN OORT F. 2009. Des sols contaminés ? in Regards croisés sur les sols urbains et périurbains, Les Cahiers des Clubs CRIN- Paris, chapitre 4, p. 98-132.

BARLES S., 1999, La ville délétère. Médecins et ingénieurs dans l'espace urbain. XVIII-XIX ${ }^{e s}$ siècles, Seyssel, Collection Milieux. Champ Vallon, $370 \mathrm{p}$.

BARLES S., 2002, Le métabolisme urbain et la question écologique, Annales de la recherche urbaine, $\mathrm{n}^{\circ} 92$ Ce qui demeure, p. 143-150.

BAXTER W., AURISICCHIO M., CHILDS P., 2017, Contaminated interaction: another barrier to circular material flows, Journal of Industrial Ecology, Volume 21, n³, p. 507-516.

BESNARD C., BRANCHU P., CHARVET R., DOUAY F., LEBEAU T., LEFEVRE G., LE GUERN C., PELFRENE A., REMY E., Guide " Qualité et usages des sols urbains : points de vigilance », POLLUSOLS, $17 \mathrm{p}$.

BLANC J., DUBOST F., LIZET B., 2015, Vivables, vivantes et vivrières : de nouveaux espoirs pour la ville ?, Revue d'ethnoécologie, ${ }^{\circ} 8$ [En ligne], mis en ligne le 04 juillet 2016.

BLANCHON D. et al., 2009, Comprendre et construire la justice environnementale, Annales de géographie, 1, n665-666, p. 35-60. DOI 10.3917/ag.665.0035

BONNEFOY S., BRAND C., 2014, Régulation politique et territorialisation du fait alimentaire : de l'agriculture à l'agri-alimentaire, Géocarrefour, vol 89, n²1-2, p. 95-103. 
BOULDING KENNETH E., 1966, The economics of the coming spaceship earth, in H. JARRETT (ed.), Environmental Quality in a Growing Economy, Baltimore, MD : Resources for the Future, Johns Hopkins University Press, p. 3-14.

CANAVESE M., REMY E., DOUAY F., BERTHIER N., 2017, Entre mémoire des sols et amnésie sociale : l'émergence de la dimension socio-politique de la qualité des sols de jardins collectifs urbains, in BOURDEAU-LEPAGE L. (dir.), Nature en ville, Désirs \& controverses, p. 88-96.

CANOLLE E., 2016, Aménagement des espaces verts parisiens : évolution des mesures de caractérisation des sols et de leur qualité. Master 2 Sciences de l'Univers, de l'Environnement et de l'Écologie, Parcours Géochimie Environnementale, UPMC, 37 p.

CENTRE D'ÉTUDES SUR LES RÉSEAUX, LES TRANSPORTS, L'URBANISME ET LES CONSTRUCTIONS PUBLIQUES, 2001, Composer avec la nature en ville, Mâcon, Edition TEC \& DOC, 375 p.

CORVOL A. (Éd.), 1994, La nature en ville XVIIe-XXe siècle. Collection : Cahier d'études Forêt, environnement et société XVIe-XXe siècle, Groupe d'histoire des forêts françaises, Centre National de la Recherche Scientifique, Institut d'Histoire Moderne et Contemporaine, 68 p.

COUSINS J.-C., NEWELL J.-P., 2015, A political-industrial ecology of water supply infrastructure for Los Angeles, Geoforum, DOI:10.1016/j.geoforum.2014.10.011.

DANIEL A.C, 2014, Les terres d'apport d'Ile-de-France, rapport Jassur.

DRON C, 2009, Résultats et enseignements des actions mises en œuvre en 2008 sur le site des « Murs à pêches » de Montreuil-sous-Bois visant à caractériser la contamination des sols par les éléments traces métalliques (ETM) et à appréhender l'impact sur la conformité réglementaire des productions maraîchères et fruitières, rapport DRIAFF.

DUCHEMIN E., 2013, Agriculture urbaine : aménager et nourrir la ville, VertigO, 389 p.

ERKMAN S., 1997, Industrial ecology: an historical view, Journal of Cleaner Production.Vol 5, No. 1-2, p. $1-10$

ERNWEIN M., SALOMON-CAVIN J., 2014, Au-delà de l'agrarisation de la ville : l'agriculture peutelle être un outil d'aménagement urbain ? Discussion à partir de l'exemple genevois, Géocarrefour, vol. 89, $n^{\circ} 1-2$, p. 31-40.

FOTI L., DUBS F., GIGNOUXA J., LATA J-C, LERCHAT- Z., MATHIEU J., NOLD F., NUNANA N., RAYNAUDA X., ABBADIE L., BAROT S., 2017, Trace element concentrations along a gradient of urban pressure in forest and lawn soils of the Paris region (France), Science of the Total Environment, Volume 598,15 novembre 2017, p. 938-948.

FROSCH R., GALLOPOULOS N., 1989, Strategies for Manufacturing, Scientific American, Septembre, vol 3, n²61, p. 94-102

GARNAUD S., MOUCHEL J.-M., CHEBBO G., THEVENOT D.R., 1999, Heavy metal concentrationsin dry and wet atmospheric deposits in Paris district: comparison with urban runoff, Science of the Total Environment, 235, p. 235-245.

GAULIN C., 1987, Horticulteurs et maraîchers parisiens de la seconde moitié du XIX ${ }^{\mathrm{e}}$ siècle à la première guerre mondiale, JATBA, Vol. 34, $\mathrm{n}^{\circ} 1$, p. 113-123.

GITTON C., 2016, Histoire de l'aménagement des espaces verts et de l'environnement urbain parisien: comprendre la contamination en éléments traces métalliques. Mémoire de master 2, GAELE Spécialité EDTS Université Paris-Sorbonne. $125 \mathrm{p}$.

GUEYMARD S., LOPEZ C., 2015, L'économie circulaire : un nouvel axe stratégique pour l'île-de-France, Note rapide, $\mathrm{n}^{\circ} 706$, IAU îdF, décembre. 
HOLLING C.S., 1973, Resilience and stability of ecological systems, Annual Review of Ecology and Systematics 4, p. 1-23.

HCSP, 2014, Détermination de nouveaux objectifs de gestion des expositions au plomb : Rapport, Collection Avis et Rapports - Haut Conseil en Santé Publique. 99 p.

JOIMEL S., CORTET J., JOLIVET C.C., SABY N., CHENOT E.D., BRANCHU P., CONSALES J.-N., LEFORT C., MOREL J.-L., SCHWARTZ C., 2016, Physico-chemical characteristics of topsoil for contrasted forest, agricultural, urban and industrial land uses in France, Science of the Total Environment, 555, p. $40-47$.

JONSSON A., SVIDEN J., 1999, 200 Years of Mercury Metabolism in Stockholm and its Impact on Aquatic Environment, communication au colloque international Nature, Society, History Long Term Dynamics of Social Metabolism, Vienne, Institute for Interdisciplinary Studies of Austrian Universities, 30 sept. -2 oct.

LARBALETRIER A., 1898, Manuel d'essais pratiques de chimie horticole, $136 \mathrm{p}$.

LARRERE C., 2009, La justice environnementale, Multitudes, /1 ( $\left.{ }^{\circ} 36\right)$, p. 156-162. DOI 10.3917/ mult.036.0156.

LATOUR B., 2006, Changer de société. Refaire de la sociologie, Paris, La Découverte, 400 p.

LESTEL L., 2002, Le plomb, in BARLES S., ed., Analyse rétrospective du système Seine, rapport de synthèse dans le cadre du programme PIREN-Seine 1998-2001, Paris, UMR CNRS 7619 Sisyphe, p. 26-30.

LEVY J.C., 2009, L'économie circulaire : l'urgence écologique? Monde en transe, Chine en transit, Presses de l'École nationale des Ponts \& Chaussées, 176 p.

LUCAS J-C., 2015, Histoire des espaces verts de Paris, Cycle de Conférences histoire des paysages, des espaces verts et des jardins. Ecole du Breuil. DEVE.

LUPTON S., 2011, Economie des déchets. Une approche institutionnaliste, Bruxelles, De Boeck, 264 p.

MANDINAUD V., 2005, La pollution des sols des champs d'épandage d'eaux usées, contrainte et/ ou ressource pour le développement durable en plaine de Bessancourt-Herblay-Pierrelaye, Développement durable et territoires. Économie, géographie, politique, droit, sociologie, Dossier 4.

MOREAU J.-G, DAVERNE J.-J., 1845, Manuel pratique de la culture maraîchère de Paris, Hachette, BNF, $386 \mathrm{p}$.

NEWELL J.-P., COUSINS J.-J., 2014, The boundaries of urban metabolism: Towards a politicalindustrial ecology, Progress in Human Geography, vol 6, n³9, p. 702-728.

PEARCE DAVID W., KERRY TURNER R., 1989, Economics of Natural Resources and the Environment ,Harvester Wheatsheaf, Hemel Hempstead, Hertfordshire, UK, 378 p. ISBN 0-7450-0202-1/0-7450-0225-0

REMY E, BRANCHU P, CANAVESE M, BERTHIER N., 2017, Les risques sanitaires liés aux jardins collectifs : l'expertise sur le sol urbain en débat, Lien Social et Politiques, n78, p. 49-69, URI: http:// id.erudit.org/iderudit/1039338ar.

ROY J.-M., 2012, Implantation des maraîchers parisiens de 1859 à 1959, Catalogue : "Savez-vous plantez les choux ?" depuis l'exposition : "Savez-vous plantez les choux? " au parc de Bagatelle du 12 juin au 4 novembre. 
SALOMON-CAVIN J., 2012, Entre ville stérile et ville fertile, l'émergence de l'agriculture urbaine en Suisse, Environnement Urbain / Urban Environment[En ligne], Vol. 6, mis en ligne le 16 septembre 2012.

ZHUY. G., REID B. J., MEHARG A. A., BANWART S. A., FU B. J. 2017, Optimizing Peri-URban Ecosystems (PURE) to re-couple urban-rural symbiosis, Science of the Total Environment 586, p. 1085-1090.

\section{NOTES}

1. Sur les controverses concernant la définition de «terres végétales ", se reporter au rapport de Anne-Cécile Daniel (2014).

2. En octobre 2015, à l'occasion de l'Exposition Universelle de Milan, une centaine de maires de grandes cités à travers le monde ont signé le Pacte de politique alimentaire urbaine de Milan.

3. Rencontres du 23 août 2017 organisées par l'Agence de l'Ecologie Urbaine de la ville de Paris «Paris se réinvente : agriculture urbaine et économie circulaire ».

4. En s'inspirant de l'idée de «construire la ville sur la ville» dans le cadre du renouvellement urbain.

5. L'ADEME définit sept composantes de l'économie circulaire : l'approvisionnement durable, l'écoconception, l'écologie industrielle et territoriale, l'économie de la fonctionnalité, la consommation responsable, l'allongement de la durée d'usage et le recyclage et la valorisation des déchets.

6. Pour le réaliser, nous avons délibérément soustrait des calculs, les bois de Vincennes et de Boulogne bien qu'ils s'apparentent à des EV. En effet, si nous considérons les bois comme des EV, leurs superficies à eux seuls (environ 1840 hectares contre 500 hectares d'EV stricto sensu) biaisent l'ensemble du graphique. Par ailleurs, tous les efforts de création de nouveaux espaces depuis une trentaine d'années (20 à 30 hectares par mandature) passeraient totalement inaperçus.

7. Ceci s'explique par le démantèlement de l'enceinte de Thiers située plus ou moins sur les boulevards des Maréchaux, en 1919 dans le cadre du déclassement de l'enceinte fortifiée de Paris.

8. Ces derniers, n'ayant pas de pelouse n'entrent pas dans le cadre de ce travail mais on fait l'objet d'études spécifiques (Canavese et al. 2017 ; Rémy et al., 2017).

9. Source : communication personnelle.

10. Ce que réactualisent les initiatives de composts urbains confectionnés à partir de feuilles ramassées sur les trottoirs qui font depuis peu l'objet d'études pour mieux connaître leur qualité. Précisons que le terme de "balayures des rues" ne désigne pas seulement le ramassage des feuilles car la ville d'hier était souillée par de nombreux déchets, comme nous le verrons plus loin.

11. L'expression " la petite culture des environs » ne désigne probablement pas la proche banlieue à cette époque mais des communes situées autrefois à l'extérieur de l'enceinte des fermiers généraux (ancienne délimitation de la ville de Paris avant 1860) comme la commune de Vaugirard, Vanves, Passy, Montrouge, Belleville, Montmartre ou encore La Villette etc., et annexées à Paris sous Napoléon III.

12. $47 \%$ des boues de station d'épuration sont épandues en agriculture, $26 \%$ sont compostées et utilisées comme amendement organique, 19\% sont incinérées et $8 \%$ sont mises en décharges (chiffres 2008 du Ministère de la transition écologique et solidaire). 
13. Cette pratique d'épandage agricole des boues est actuellement encadrée par la réglementation.

14. Entretien réalisé par C. Gitton (op. cit.).

15. Précisons que jusqu'à la fin des années 1940, le plomb dans les peintures est la source principale de l'intoxication. Aujourd'hui d'autres sources environnementales sont mises en avant, notamment les sols.

16. Entretien réalisé par C. Gitton (op. cit.)

17. A noter aussi que certains marâichers partaient avec leurs terres. Ces pratiques étaient-elles généralisées ? À quelle période ? Ce qui souligne la difficulté de retracer précisément le parcours de ces terres au cours du temps.

18. Les terres destinées au maraîchage sont généralement à dominante sableuse ; ce qui améliore leur précocité (réchauffement rapide dû à leur faible rétention d'eau) et facilite le travail intensif (nombreuses rotations culturales). Dans l'ouvrage de S. Barles (op.cit.), on comprend que les ingénieurs de la Ville de Paris avaient pour objectif d'éliminer de grands volumes d'eaux usées. Il s'agissait d'une technique dite de " colmatage des sols », ce qui ne pouvait se concevoir que sur des sols sableux typiques du maraîchage.

19. 1950 étant la date qui semble marquer le changement d'origine de la terre, et 1990 celle à partir de laquelle les terres d'apport ont été contrôlées par la Ville de Paris sur le plan agronomique. Les ETM sont contrôlés depuis 2015.

20. Le mélange de terre est une pratique assez répandue dans les jardins. Pour améliorer le comportement des «vieilles terres" (renforcement de la structure, dilution des réserves organiques et minérales) et réduire les coûts liés à une évacuation complète, il est procédé à un simple rechargement de la parcelle, suivi d'un brassage avec le matériau en place.

21. Il est à noter qu'il manque encore aujourd'hui une traçabilité des terres végétales rapportées. 22. Des controverses apparaissent alors sur la capacité du sol urbain à accueillir ou non des cultures à vocation alimentaire, comme l'a montré l'analyse comparée du guide de Toronto et celle du protocole mis en œuvre par la ville de Paris. Le débat porte sur les différentes façons d'évaluer le degré de contamination des sols urbains et de définir leurs usages (Rémy et al., op.cit .).

23. En termes de gestion des déchets par les sols, des choix techniques et politiques ont déjà eu des conséquences désastreuses : le 31 mai 1999 puis le 31 mars 2000, sur avis du CSHPF (Conseil Supérieur d'Hygiène Publique de France), les préfets des Yvelines et du Val d'Oise ont pris des arrêtés d'interdiction de mise sur le marché de cultures maraîchères (légumes et plantes aromatiques) produites sur les plaines de Pierrelaye, Bessancourt et d'Achères. Cette interdiction concernait une trentaine de maraîchers.

24. Même si des contrats de recherche récents y contribuent comme POTEX, REPJAR, POLLUSOLS, JASSUR et un guide dédié à cette question (2017) à destination du grand public.

\section{RÉSUMÉS}

Dans le cadre de la prévention du saturnisme infantile, le Haut Conseil de la Santé Publique (HCSP) a fixé en 2014 des seuils de gestion liés aux concentrations en plomb dans les sols. En effet, au regard des risques liés à l'ingestion de particules de terre liée au portée main-bouche pour les enfants de moins de 6 ans, un seuil de vigilance de $100 \mathrm{mg}$ de plomb par kg de terre a été 
fixé dans les sols des espaces verts. Suite à la parution de ces nouveaux objectifs de gestion, la Ville de Paris a amorcé un travail de caractérisation des sols de ses 500 ha d'espaces verts (EV). Ainsi, des analyses d'éléments traces métalliques (ETM), d'hydrocarbures et de paramètres agronomiques ont été réalisées sur 102 premiers EV. Ces mesures de qualité des sols, confrontées à la reconstitution de l'histoire des espaces verts, ont permis de dresser un certain nombre d'hypothèses quant à l'origine des terres végétales qui ont été utilisées depuis 200 ans. Alors que les efforts pour passer à une économie circulaire aboutissent à privilégier les circuits courts et l'approvisionnement local, il s'agit de s'interroger sur les pollutions potentielles des terres d'apports qui ont transité et qui transitent encore de la proche banlieue vers les centres urbains pour l'aménagement des espaces verts accueillant de plus en plus des potagers urbains. Dans ce contexte, il n'est pas inutile de réinterroger les principes et les rouages complexes de l'économie circulaire appliquée aux flux de terres végétales dans une démarche à la fois historique et spatiale.

As part of the measures taken to prevent infantile saturnism, the French Higher Council for Public Health (Haut Conseil de la Santé Publique - HCSP) set thresholds values in 2014 for lead concentrations in the soil. Considering the risks linked to the ingestion of particles of soil by children under the age of six, who tend to put their hands in their mouth, a vigilance threshold value of $100 \mathrm{mg}$ of lead per kilogram of soil was set for green spaces. Following the publication of these new objectives, the City of Paris initiated a program intended to characterize the soil of its 102 green spaces representing 500 ha. Analyses of metallic trace elements, of hydrocarbons and of agronomic parameters were carried out to assess soil quality. Related to the history of the green spaces, they provided the basis for several hypotheses on the origins of the topsoil that has been used in vegetable gardens over the past 200 years. Considering the efforts made to shift to a circular economy, that favour short circuits and local supplies, this paper critically examines the potential pollution of soils which move from the close suburbs to the green spaces of urban centres, where more and more vegetable gardens are developed. In this context, it is interesting to re-examine the principles and complex workings of the circular economy applied to the evolution of vegetable gardens from a historical and spatial point of view.

\section{INDEX}

Keywords : green spaces, cities, soil, circular economy, pollution, trace elements, lead, sewage sludge

Mots-clés : espaces verts, ville, sols, économie circulaire, pollution, éléments en trace, plomb, boues d'épandage

\section{AUTEURS}

\section{CLÉMENT GITTON}

clement_gitton@yahoo.fr

\section{YOANN VERGER}

UMR 1402 ECOSYS, INRA, AgroParisTech, Université Paris-Saclay, Bâtiment Bioclimatologie, route de la Ferme, 78850 Grignon. yoann.verger@inra.fr 


\section{FLORENCE BRONDEAU}

UMR 8185 ENeC, 28 rue Serpente, 75006, Institut de Géographie Université Paris-Sorbonne, 191 rue Saint-Jacques 75005 Paris. Florence.Brondeau@paris-sorbonne.fr

\section{RONALD CHARVET}

Pôle pollution des sols et ICPE, Division Impact Santé Environnement, Agence d'Écologie Urbaine, Direction des Espaces Verts et de l'Environnement, Ville de Paris, 103 Av de France 75013 Paris ronald.charvet@paris.fr

\section{FRANÇOIS NOLD}

Laboratoire d'agronomie, Service des Sciences et Techniques du Végétal, Direction des Espaces Verts et de l'Environnement - Ville de Paris, Chênaie du Parc Floral, Route de la Pyramide 75012 Paris. Francois.nold@paris.fr

\section{PHILIPPE BRANCHU}

Cerema Ile-de-France, 12 Rue Léon Teisserenc de Bort, 78190 Trappes,

Philippe.Brachu@cerema.fr

\section{FRANCIS DOUAY}

LGCgE, ISA Lille Yncréa Hauts-de-France, 48 boulevard Vauban 59046 Lille Cedex, francis.douay@yncrea.fr

\section{ISABELLE LAMY}

UMR1402 ECOSYS, INRA, AgroParisTech, Université Paris-Saclay, Pôle Ecotoxicologie, RD10 78026 Versailles Cedex. isabelle.lamy@inra.fr

\section{CHRISTIAN MOUGIN}

UMR1402 ECOSYS, INRA, AgroParisTech, Université, Paris-Saclay, Pôle Ecotoxicologie, RD10 78026 Versailles Cedex. christian.mougin@inra.fr

\section{CAROLINE PETIT}

UMR 1048 SAD-APT, INRA, AgroParisTech, Université Paris-Saclay, 16 rue Claude Bernard, 75231 Paris cedex 05. caroline.petit@agroparistech.fr

\section{ELISABETH RÉMY}

UMR 1048 SAD-APT, INRA, AgroParisTech Sad-Apt, Université Paris-Saclay, 16 rue Claude Bernard, 75231 Paris cedex 05. elisabeth.remy@agroparistech.fr 